\title{
Superfield realization of hidden $R$-symmetry in extended supersymmetric gauge theories and its applications
}

\author{
I.L. Buchbinder, ${ }^{a, b}$ E.A. Ivanov ${ }^{c}$ and V.A. Ivanovskiy ${ }^{d}$ \\ ${ }^{a}$ Department of Theoretical Physics, Tomsk State Pedagogical University, \\ 634061, Tomsk, Russia \\ ${ }^{b}$ National Research Tomsk State University, \\ Tomsk, Russia \\ ${ }^{c}$ Bogoliubov Laboratory of Theoretical Physics, JINR, \\ 141980 Dubna, Moscow region, Russia \\ ${ }^{d}$ Moscow Institute of Physics and Technology, \\ 1417000 Dolgoprudny, Russia \\ E-mail: joseph@tspu.edu.ru, eivanov@theor.jinr.ru, \\ ivanovskiy.va@phystech.edu
}

ABSTRACT: We present the explicit superfield realizations of the hidden SU(4) and $O(5)$ $R$-symmetries in $4 D, \mathcal{N}=4$ and $5 D, \mathcal{N}=2$ supersymmetric Yang-Mills theories in the harmonic superspace approach. The $R$-symmetry transformations are constructed and their algebraic structure is studied. It is shown that such transformations are consistent with both manifest and hidden supersymmetry transformations. These symmetries can serve as an alternative tool for constructing the relevant complete low-energy superfield effective actions determined earlier from the hidden supersymmetry considerations.

KeYwORDS: Extended Supersymmetry, Superspaces

ARXiv EPRINT: 2001.01649 


\section{Contents}

1 Introduction 1

$24 D, \mathcal{N}=4$ SYM theory 3

2.1 A sketch of $\mathcal{N}=2$ harmonic superspace 3

2.2 Classical action of $\mathcal{N}=4 \mathrm{SYM}$

$2.3 R$-symmetry transformations $\quad 5$

2.4 Effective action 8

$\begin{array}{ll}2.5 & \text { Superconformal invariance of the effective action } \\ \end{array}$

$35 D, \mathcal{N}=2$ SYM theory $\quad 12$

$\begin{array}{lll}3.1 & \text { Classical action } & 13\end{array}$

$\begin{array}{lll}3.2 & R \text {-symmetry transformations } & 13\end{array}$

$\begin{array}{lll}3.3 & \text { Effective action } & 15\end{array}$

$\begin{array}{lll}4 & \text { Summary } & 18\end{array}$

A Commutators of $R$-symmetry transformations in $4 D, \mathcal{N}=4 \mathrm{SYM}$ theory 19

B Commutators of $R$-symmetry transformations in $5 D, \mathcal{N}=2$ SYM theory 21

\section{Introduction}

Classical and quantum supersymmetric Yang-Mills (SYM) theories with 16 supercharges in diverse dimensions play an important role in the modern field theory(see, e.g., [1]). The main source of interest in them is the property that they describe low-energy limit of some brane-like compactifications of type II string theory and thereby provide a bridge between superstring theory and supersymmetric field theory. Most interesting and elaborated are $4 D, \mathcal{N}=4,5 D, \mathcal{N}=2$ and $6 D, \mathcal{N}=(1,1)$ SYM theories. Some of these models admit superfield formulations with half of the underlying supersymmetry being manifest and offshell, namely, with $4 D, \mathcal{N}=2,5 D, \mathcal{N}=1$ and $6 D, \mathcal{N}=(1,0)$ off-shell supersymmetries. These formulations were constructed within the relevant harmonic superspaces $[2,3]$.

In such formulations, the second half of the total supersymmetry is realized on the basic superfields of the theory as a hidden (or implicit) supersymmetry which forms a closed Lie bracket structure with the manifest supersymmetry only on shell. While inspecting the superfield quantum effective actions, the role of this hidden half of supersymmetry turns out to be very restrictive: requiring the effective action to enjoy such a supersymmetry fixes, in most known cases, its structure up to an overall coefficient which should further be explicitly calculated from the relevant superfield quantum perturbation theory. A review of this approach is given in refs. [4-6]. 
The basic example of applying such a strategy for constructing the quantum effective action is provided by $4 D, \mathcal{N}=4 \mathrm{SYM}$ effective action which was constructed in [7] as a hypermultiplet completion of the non-holomorphic $\mathcal{N}=2$ gauge superfield potential found in [8]. Later on, the same effective action was reproduced in various harmonic superspaces [5]. These works revealed a correspondence between the $\mathcal{N}=4 \mathrm{SYM}$ lowenergy effective action and the leading terms in the effective action of D3 brane on the $A d S^{5} \times S^{5}$ background. One more example is $5 D, \mathcal{N}=2 \mathrm{SYM}$ theory. Its low-energy effective action was constructed as a hypermultiplet completion of the $5 D, \mathcal{N}=1 \mathrm{SYM}$ effective action [9].

In this parer we propose another way to determine the low-energy effective actions of $4 D, \mathcal{N}=4$ and $5 D, \mathcal{N}=2 \mathrm{SYM}$ theories in harmonic superspace. It is based upon exploiting a hidden bosonic $R$-symmetry of these theories instead of hidden supersymmetry. To be more precise, we suggest a new possibility to construct the superspace functionals depending on all fields of the corresponding supermultiplet, beginning with a functional which involves only part of such fields. The point is that the supersymmetry algebra possesses the automorphism group which is called the $R$-symmetry group. We will show that such $R$-symmetry can be realized directly on harmonic superfields. While some subgroups of $R$-symmetry are realized linearly and manifestly, the rest of its transformations proves to possess a highly non-trivial realization mixing all fields of the extended supermultiplet. As a result, we gain a possibility to impose the condition of invariance under the total $R$-symmetry on a superspace functional in order to specify its dependence on all fields of such a supermultiplet.

In the harmonic superspace formulation, the full multiplets of $4 D, \mathcal{N}=4$ or $5 D$, $\mathcal{N}=2 \mathrm{SYM}$ theories consist of the gauge vector multiplet and the hypermultiplet. Not only half of $4 D, \mathcal{N}=4$ and $5 D, \mathcal{N}=2$ supersymmetries is realized in an implicit way, but also that part of the total $R$-symmetries of these theories, viz., of $\mathrm{SU}(4)$ and $\mathrm{SO}(5)$, which mixes the hidden and manifest supersymmetry transformations. It is also realized by some implicit transformations. In this paper we find the precise form of the hidden $R$-symmetry transformations which extend the manifest $R$-symmetry groups, namely $\mathrm{U}(2) \times \mathrm{SU}(2)$ and $\mathrm{SU}(2) \times \mathrm{SU}(2)$, to $\mathrm{SO}(6)$ or $\mathrm{SO}(5)$, respectively.

Although the low-energy effective action might be found by direct quantum computations in harmonic superspace or by using the hidden supersymmetry transformations, we determine it here in a different way. Namely, we construct the hypermultiplet completions of $4 D, \mathcal{N}=2$ and $5 D, \mathcal{N}=1$ leading terms by imposing the requirement of full $R$-symmetry invariance. The effective action corresponds to the Coulomb branch, with the gauge group being broken to some abelian subgroup. For simplicity we concentrate on $\mathrm{SU}(2)$ gauge group broken to $\mathrm{U}(1)$ and consider, as usual in the Coulomb phase, only that part of the effective action which depends on the fields of massless $\mathrm{U}(1) \mathcal{N}=2$ gauge multiplet and its neutral hypermultiplet partner forming together $4 D, \mathcal{N}=4$ or $5 D, \mathcal{N}=2$ abelian $\mathrm{U}(1)$ gauge multiplets.

In addition, we explicitly show that the $4 D, \mathcal{N}=4 \mathrm{SYM}$ effective action respects superconformal invariance. 


\section{$24 D, \mathcal{N}=4$ SYM theory}

In this section we present the superfield realization of the hidden part of the $R$-symmetry transformations for $4 D, \mathcal{N}=4 \mathrm{SYM}$ theory with the gauge group $\mathrm{SU}(2)$ and construct the low-energy effective action. As an additional exercise, we show its superconformal invariance.

\subsection{A sketch of $\mathcal{N}=2$ harmonic superspace}

In our presentation we basically follow the notation and conventions of refs. [3, 5]. Some important notions of the harmonic superspace approach are briefly outlined below.

The standard $4 D, \mathcal{N}=2$ superspace is parametrized by the coordinates

$$
z^{M}=\left(x^{m}, \theta_{i}^{\alpha}, \bar{\theta}^{\dot{\alpha} i}\right),
$$

where $x^{m}, m=0,1,2,3$, are the Minkowski space coordinates and $\theta_{i}^{\alpha}, \bar{\theta}^{\dot{\alpha} i}, i=1,2$, $\alpha, \dot{\alpha}=1,2$, are the anticommuting Grassmann coordinates.

One can add, to this set of coordinates, the harmonics $u^{ \pm i}\left(u_{i}^{-}=\left(u^{+i}\right)^{*}, u^{+i} u_{i}^{-}=1\right)$ which describe the "harmonic sphere" $\mathrm{SU}(2)_{R} / \mathrm{U}(1)$, where $\mathrm{SU}(2)_{R}$ is the $R$-symmetry group acting on the doublet indices $i, k$. The $4 D, \mathcal{N}=2$ harmonic superspace in the central basis is defined as the enlarged coordinate set

$$
Z=(z, u)=\left(x^{m}, \theta_{i}^{\alpha}, \bar{\theta}^{\dot{\alpha} i}, u^{ \pm i}\right) .
$$

In the analytic basis it is parametrized by the coordinates

$$
\begin{aligned}
Z_{(a n)} & =\left(x_{(a n)}^{m}, \theta_{\alpha}^{ \pm}, \bar{\theta}_{\dot{\alpha}}^{ \pm}, u^{ \pm i}\right), \\
x_{a n}^{m} & =x^{m}-2 i \theta^{(i} \sigma^{m} \bar{\theta}^{j)} u_{i}^{+} u_{j}^{-}, \quad \theta_{\alpha}^{ \pm}=u_{i}^{ \pm} \theta_{\alpha}^{i}, \quad \bar{\theta}_{\dot{\alpha}}^{ \pm}=u_{i}^{ \pm} \bar{\theta}_{\dot{\alpha}}^{i} .
\end{aligned}
$$

The most important feature of the analytic basis consists in that the set of coordinates

$$
\zeta=\left(x_{(a n)}^{m}, \theta_{\alpha}^{+}, \bar{\theta}_{\dot{\alpha}}^{+}, u^{ \pm i}\right),
$$

involving only half of the original Grassmann coordinates, forms a subspace closed under the $4 D, \mathcal{N}=2$, supersymmetry transformations. The set (2.5) represents what is called the "harmonic analytic superspace".

The important ingredients of the harmonic superspace approach are the spinor and harmonic derivatives. In the analytic basis, they are expressed as

$$
\begin{array}{rlrl}
D_{\alpha}^{+} & =\frac{\partial}{\partial \theta^{-\alpha}}, & \bar{D}_{\dot{\alpha}}^{+}=\frac{\partial}{\partial \bar{\theta}^{-\dot{\alpha}}}, \\
D_{\alpha}^{-}=-\frac{\partial}{\partial \theta^{+\alpha}}+2 i \bar{\theta}^{-\dot{\alpha}} \partial_{\alpha \dot{\alpha}}, & \bar{D}_{\dot{\alpha}}^{-}=-\frac{\partial}{\partial \theta^{+\dot{\alpha}}}+2 i \theta^{-\alpha} \partial_{\alpha \dot{\alpha}}, \\
D^{++}=u^{+i} \frac{\partial}{\partial u^{-i}}-2 i \theta^{+\alpha} \bar{\theta}^{+\dot{\alpha}} \partial_{\alpha \dot{\alpha}}+\theta^{+\alpha} \frac{\partial}{\partial \theta^{-\alpha}}+\bar{\theta}^{+\dot{\alpha}} \frac{\partial}{\partial \bar{\theta}^{-\dot{\alpha}}}, \\
D^{--}=u^{-i} \frac{\partial}{\partial u^{+i}}-2 i \theta^{-\alpha} \bar{\theta}^{-\dot{\alpha}} \partial_{\alpha \dot{\alpha}}+\theta^{-\alpha} \frac{\partial}{\partial \theta^{+\alpha}}+\bar{\theta}^{-\dot{\alpha}} \frac{\partial}{\partial \bar{\theta}^{+\dot{\alpha}}} .
\end{array}
$$


The harmonic derivatives $D^{ \pm \pm}$, together with the harmonic U(1) charge operator

$$
D^{0}=u^{+i} \frac{\partial}{\partial u^{+i}}-u^{-i} \frac{\partial}{\partial u^{-i}}+\theta^{+\alpha} \frac{\partial}{\partial \theta^{+\alpha}}+\bar{\theta}^{+\dot{\alpha}} \frac{\partial}{\partial \bar{\theta}^{+\dot{\alpha}}}-\theta^{-\alpha} \frac{\partial}{\partial \theta^{-\alpha}}-\bar{\theta}^{-\dot{\alpha}} \frac{\partial}{\partial \bar{\theta}^{-\dot{\alpha}}},
$$

form an SU(2) algebra,

$$
\left[D^{++}, D^{--}\right]=D^{0}, \quad\left[D^{0}, D^{ \pm \pm}\right]= \pm 2 D^{ \pm \pm} .
$$

The harmonic superfields (as well as the harmonic projections of the spinor covariant derivatives) carry a definite integer harmonic $\mathrm{U}(1)$ charge, $D^{0} \Phi^{q}(Z)=q \Phi^{q}(Z)$, $\left[D^{0}, D_{\alpha, \dot{\alpha}}^{ \pm}\right]= \pm D_{\alpha, \dot{\alpha}}^{ \pm}$. This harmonic charge is assumed to be strictly preserved in any superfield action defined on the superspaces (2.3) or (2.5).

The "shortness" of the spinor derivatives $D_{\alpha}^{+}, \bar{D}_{\dot{\alpha}}^{+}$in (2.6) reflects the existence of the analytic harmonic subspace (2.5) in the general harmonic superspace (2.3): one can define an analytic $\mathcal{N}=2$ superfield by imposing the proper covariant "Grassmann analyticity" constraints on a general harmonic superfield, viz., $D_{\alpha}^{+} \Phi^{q}(Z)=\bar{D}_{\dot{\alpha}}^{+} \Phi^{q}(Z)=0 \Rightarrow$ $\Phi^{q}(Z)=\phi^{q}(\zeta)$. The harmonic derivative $D^{++}$commutes with these spinor derivatives and so preserves the Grassmann harmonic analyticity: $D^{++} \Phi^{q}(Z)$ is an analytic superfield if $\Phi^{q}(Z)$ is.

\subsection{Classical action of $\mathcal{N}=4 \mathrm{SYM}$}

When formulated in $\mathcal{N}=2$ harmonic superspace, $\mathcal{N}=4$ vector gauge multiplet can be viewed as a "direct sum" of the gauge $\mathcal{N}=2$ multiplet and the hypermultiplet described, respectively, by the analytic superfields $V^{++}(\zeta)$ and $q_{a}^{+}(\zeta)=\left(q^{+}(\zeta),-\tilde{q}^{+}(\zeta)\right)$, where "tilde" means some generalized complex conjugation [3]. Both these multiplets belong to the same adjoint representation of the gauge group. The $\mathcal{N}=2$ gauge multiplet $V^{++}$is described dy the classical action [10]

$$
S_{\mathrm{SYM}}^{\mathcal{N}=2}=\frac{1}{2} \sum_{n=2}^{\infty} \operatorname{tr} \frac{(-i)^{n}}{n} \int d^{12} z d u_{1} \ldots d u_{n} \frac{V^{++}\left(z, u_{1}\right) \ldots V^{++}\left(z, u_{n}\right)}{\left(u_{1}^{+} u_{2}^{+}\right) \ldots\left(u_{n}^{+} u_{1}^{+}\right)}
$$

where integration goes over the total harmonic superspace and the harmonic distributions $1 /\left(u_{1}^{+} u_{2}^{+}\right), \cdots$ are defined in [3].

This action yields the following equations of motion

$$
\left(D^{+}\right)^{2} W=0, \quad\left(\bar{D}^{+}\right)^{2} \bar{W}=0,
$$

where $\left(D^{+}\right)^{2}=D^{+\alpha} D_{\alpha}^{+},\left(\bar{D}^{+}\right)^{2}=\bar{D}_{\dot{\alpha}}^{+} \bar{D}^{+\dot{\alpha}}$ and $D_{\alpha}^{+}, \bar{D}_{\dot{\alpha}}^{+}$are the harmonic plus-projection of the gauge-covariant spinor derivatives in the so called " $\lambda$ " frame, in which these derivatives require no gauge connection terms and coincide with their flat counterparts defined in (2.6), $W$ and $\bar{W}$ are chiral and antichiral gauge superfield strengths. The latter can de expressed in terms of the non-analytic harmonic gauge connection $V^{--}$,

$$
W=-\frac{1}{4}\left(\bar{D}^{+}\right)^{2} V^{--}, \quad \bar{W}=-\frac{1}{4}\left(D^{+}\right)^{2} V^{--},
$$


where $V^{--}$is related to $V^{++}$by the harmonic flatness condition

$$
D^{--} V^{++}-D^{++} V^{--}+i\left[V^{++}, V^{--}\right]=0 .
$$

The classical action for the analytic hypermultiplet in the adjoint representation reads $[2]$

$$
S_{q}=\frac{1}{2} \operatorname{tr} \int d \zeta^{-4} q_{a}^{+} \nabla^{++} q^{+a}=\frac{1}{2} \operatorname{tr} \int d \zeta^{-4} q_{a}^{+}\left(D^{++} q^{+a}+i\left[V^{++}, q^{+a}\right]\right)
$$

where $d \zeta^{-4}$ is the measure of integration over the analytic harmonic superspace. This action is invariant under an extra $\mathrm{SU}(2)_{\mathrm{PG}}$ symmetry transforming $q^{+a}$ as a doublet. Both actions (2.8) and (2.12) are invariant under the standard linear automorphism group $\mathrm{SU}(2)_{R}$ which rotates the doublet indices of the harmonic variables. In addition, both actions are invariant under the separate $R$-symmetry $\mathrm{U}(1)_{R}$ which transforms $\theta^{ \pm}$and $\bar{\theta}^{ \pm}$by the conjugated phase factors. Correspondingly, $W$ and $\bar{W}$ defined in (2.10) are also transformed by the appropriate mutually conjugated phase factors, $q^{+a}$ is the $\mathrm{U}(1)_{R}$ singlet.

The action of $\mathcal{N}=4 \mathrm{SYM}$ theory in $\mathcal{N}=2$ harmonic superspace is the sum of the actions (2.8) and (2.12),

$$
S_{\mathrm{SYM}}^{\mathcal{N}=4}=S_{\mathrm{SYM}}^{\mathcal{N}=2}+S_{q} .
$$

The total action is invariant under the following hidden $\mathcal{N}=2$ supersymmetry transformations which complement the manifest $\mathcal{N}=2$ supersymmetry to the full $\mathcal{N}=4$ supersymmetry

$$
\delta V^{++}=\left[\epsilon^{a \alpha} \theta_{\alpha}^{+}-\bar{\epsilon}_{\dot{\alpha}}^{a} \bar{\theta}^{+\dot{\alpha}}\right] q_{a}^{+}, \quad \delta q_{a}^{+}=-\frac{1}{32}\left(D^{+}\right)^{2}\left(\bar{D}^{+}\right)^{2}\left[\epsilon_{a}^{\alpha} \theta_{\alpha}^{-} V^{--}+\bar{\epsilon}_{a \dot{\alpha}} \bar{\theta}^{-\dot{\alpha}} V^{--}\right],
$$

with $\bar{\epsilon}_{a \dot{\alpha}}$ and $\epsilon_{a}^{\alpha}$ as new anticommuting parameters. Though checking the invariance of the action does not require the use of the classical equations of motion, the algebra of these transformations is closed modulo terms proportional to the equations of motion. Therefore, in this formulation only the manifest $\mathcal{N}=2$ supersymmetry is off-shell closed.

\section{$2.3 \quad R$-symmetry transformations}

We define the additional $R$-symmetry transformations of the gauge and hypermultiplet harmonic superfields as follows

$$
\begin{aligned}
\delta V^{++} & =\left[\lambda^{-a}\left(\theta^{+}\right)^{2}+\bar{\lambda}^{-a}\left(\bar{\theta}^{+}\right)^{2}\right] q_{a}^{+}, \\
\delta q_{a}^{+} & =\frac{\left(D^{+}\right)^{2}\left(\bar{D}^{+}\right)^{2}}{64}\left[\lambda_{a}^{+}\left(\theta^{-}\right)^{2} V^{--}-2 \lambda_{a}^{-} \theta^{+\alpha} \theta_{\alpha}^{-} V^{--}+\bar{\lambda}_{a}^{+}\left(\bar{\theta}^{-}\right)^{2} V^{--}-2 \bar{\lambda}_{a}^{-} \bar{\theta}_{\dot{\alpha}}^{+} \bar{\theta}^{-\dot{\alpha}} V^{--}\right],
\end{aligned}
$$

where $\lambda^{-a}=\lambda^{i a} u_{i}^{-}, \bar{\lambda}^{-a}=\bar{\lambda}^{i a} u_{i}^{-}, \overline{\lambda_{i a}}=\bar{\lambda}^{i a}, \lambda^{i a}$ are the commuting dimensionless complex parameters. These transformations extend the $R$-symmetry group from $\mathrm{SU}(2)_{R} \times \mathrm{SU}(2)_{\mathrm{PG}}$ to $\mathrm{SU}(4)$. The direct check shows that the action (2.13) is off-shell invariant under the transformations (2.15). The form of (2.15) is almost uniquely specified by the dimensionality and analyticity reasonings, together with requiring both sides to have the same 
harmonic U(1) charges. To avoid a possible confusion, we point out that the superfields in (2.15) are not subject to any on-shell conditions which should be taken into account only when inspecting the closure properties of these transformations (see below).

Further in this section we consider the case of abelian gauge group, since the effective action we will deal with depends only on the superfields of the abelian U(1) gauge multiplet. The equations of motion implied by the action (2.13) read

$$
D^{++} q_{a}^{+}=0, \quad\left(D^{+}\right)^{2} W=0, \quad\left(\bar{D}^{+}\right)^{2} \bar{W}=0 .
$$

In addition, the hypermultiplet $q_{a}^{+}$obeys the off-shell analyticity constraints

$$
D_{\alpha}^{+} q_{a}^{+}=0, \quad \bar{D}_{\dot{\alpha}}^{+} q_{a}^{+}=0 .
$$

The superfield strengths $W, \bar{W}$ are chiral and antichiral

$$
\bar{D}_{\dot{\alpha}}^{ \pm} W=0, \quad D_{\alpha}^{ \pm} \bar{W}=0,
$$

and they satisfy the off-shell constraints

$$
D^{ \pm \pm} W=0, \quad D^{ \pm \pm} \bar{W}=0,
$$

which follow from the harmonic flatness condition (2.11) and the analyticity of $V^{++}$.

When superfields $W, \bar{W}$ and $q_{a}^{+}$obey the on-shell constraints (2.16), the transformations of hidden $\mathcal{N}=2$ supersymmetry (2.14) are simplified to

$$
\begin{aligned}
\delta W & =\frac{1}{2} \bar{\epsilon}^{\dot{\alpha} a} \bar{D}_{\dot{\alpha}}^{-} q_{a}^{+}, & \delta \bar{W} & =\frac{1}{2} \epsilon^{\alpha a} D_{\alpha}^{-} q_{a}^{+}, \\
\delta q_{a}^{+} & =\frac{1}{4}\left(\epsilon_{a}^{\alpha} D_{\alpha}^{+} W+\bar{\epsilon}_{a}^{\dot{\alpha}} \bar{D}_{\dot{\alpha}}^{+} \bar{W}\right), & \delta q_{a}^{-} & =\frac{1}{4}\left(\epsilon_{a}^{\alpha} D_{\alpha}^{-} W+\bar{\epsilon}_{a}^{\dot{\alpha}} \bar{D}_{\dot{\alpha}}^{-} \bar{W}\right),
\end{aligned}
$$

where $q^{-a}=D^{--} q^{+a}$. In this case the $R$-symmetry transformations (2.15) are also simplified:

$$
\begin{aligned}
\delta q_{a}^{+} & =\frac{1}{4}\left(\lambda_{a}^{+} W-\lambda_{a}^{+} \theta^{-\alpha} D_{\alpha}^{+} W+\lambda_{a}^{-} \theta^{+\alpha} D_{\alpha}^{+} W+\bar{\lambda}_{a}^{+} \bar{W}-\bar{\lambda}_{a}^{+} \bar{\theta}^{-\dot{\alpha}} \bar{D}_{\dot{\alpha}}^{+} \bar{W}+\bar{\lambda}_{a}^{-} \bar{\theta}^{+\dot{\alpha}} \bar{D}_{\dot{\alpha}}^{+} \bar{W}\right), \\
\delta \bar{W} & =\frac{1}{2}\left(\lambda^{-a} q_{a}^{+}-\lambda^{+a} q_{a}^{-}-\lambda^{-a} \theta^{+\alpha} D_{\alpha}^{+} q_{a}^{-}+\lambda^{+a} \theta^{-\alpha} D_{\alpha}^{+} q_{a}^{-}\right), \\
\delta W & =\frac{1}{2}\left(\bar{\lambda}^{-a} q_{a}^{+}-\bar{\lambda}^{+a} q_{a}^{-}-\bar{\lambda}^{-a} \bar{\theta}^{+\dot{\alpha}} \bar{D}_{\dot{\alpha}}^{+} q_{a}^{-}+\bar{\lambda}^{+a} \bar{\theta}^{-\dot{\alpha}} \bar{D}_{\dot{\alpha}}^{+} q_{a}^{-}\right) .
\end{aligned}
$$

One may verify that the commutators of the $R$-symmetry transformations (2.21) with the manifest and hidden supersymmetry transformations give the consistent results. The variation of general superfield under the manifest supersymmetry reads

$$
\hat{\delta} \Phi=-\epsilon^{+\alpha} \frac{\partial \Phi}{\partial \theta^{+\alpha}}-\epsilon^{-\alpha} \frac{\partial \Phi}{\partial \theta^{-\alpha}}-\bar{\epsilon}_{\dot{\alpha}}^{+} \frac{\partial \Phi}{\partial \bar{\theta}_{\dot{\alpha}}^{+}}-\bar{\epsilon}_{\dot{\alpha}}^{-} \frac{\partial \Phi}{\partial \bar{\theta}_{\dot{\alpha}}^{-}}+2 i\left(\epsilon^{-\alpha} \bar{\theta}^{+\dot{\alpha}}+\theta^{+\alpha} \bar{\epsilon}^{-\dot{\alpha}}\right) \partial_{\alpha \dot{\alpha}} \Phi .
$$


Let us first consider the commutators of the hidden supersymmetry transformations (2.20) with the $R$-symmetry transformations (2.21). One can show, by a direct computation, that

$$
\begin{aligned}
&\left(\delta_{\lambda} \delta_{\epsilon}-\delta_{\epsilon} \delta_{\lambda}\right) q^{+a}=\frac{1}{8}\left[-\bar{\lambda}_{c}^{+} \epsilon^{\alpha c} \frac{\partial q^{+a}}{\partial \theta^{+\alpha}}-\lambda_{c}^{+} \bar{\epsilon}^{\dot{\alpha} c} \frac{\partial q^{+a}}{\partial \bar{\theta}^{+\dot{\alpha}}}\right.\left.+2 i\left(\bar{\lambda}_{c}^{-} \epsilon^{\alpha c} \bar{\theta}^{+\dot{\beta}}+\theta^{+\alpha} \lambda_{c}^{-} \bar{\epsilon}^{\dot{\beta} c}\right) \partial_{\alpha \dot{\beta}} q^{+a}\right]=\frac{1}{8} \hat{\delta}_{\bar{\lambda}_{c}^{+} \epsilon^{\alpha c} q^{+a},} \\
&\left(\delta_{\lambda} \delta_{\epsilon}-\delta_{\epsilon} \delta_{\lambda}\right) W=\frac{1}{8}\left[-\bar{\lambda}_{c}^{+} \epsilon^{\alpha c} \frac{\partial W}{\partial \theta^{+\alpha}}-\bar{\lambda}_{c}^{-} \epsilon^{\alpha c} \frac{\partial W}{\partial \theta^{-\alpha}}-\lambda_{c}^{-} \bar{\epsilon}^{\dot{\alpha} c} \frac{\partial W}{\partial \bar{\theta}^{-\dot{\alpha}}}\right. \\
&\left.+2 i\left(\bar{\lambda}_{c}^{-} \epsilon^{\alpha c} \bar{\theta}^{+\dot{\beta}}+\theta^{+\alpha} \lambda_{c}^{-} \bar{\epsilon}^{\dot{\beta} c}\right) \partial_{\alpha \dot{\beta}} W\right]=\frac{1}{8} \hat{\delta}_{\bar{\lambda}_{c}^{+} \epsilon^{\alpha c} W}, \\
&\left(\delta_{\lambda} \delta_{\epsilon}-\delta_{\epsilon} \delta_{\lambda}\right) \bar{W}=\frac{1}{8}\left[-\bar{\lambda}_{c}^{-} \epsilon^{\alpha c} \frac{\partial \bar{W}}{\partial \theta^{-\alpha}}-\lambda_{c}^{-} \bar{\epsilon}^{\dot{\alpha} c} \frac{\partial \bar{W}}{\partial \bar{\theta}^{-\dot{\alpha}}}-\lambda_{c}^{+} \bar{\epsilon}^{\dot{\alpha} c} \frac{\partial \bar{W}}{\partial \bar{\theta}^{+\dot{\alpha}}}\right. \\
&\left.+2 i\left(\bar{\lambda}_{c}^{-} \epsilon^{\alpha c} \bar{\theta}^{+\dot{\beta}}+\theta^{+\alpha} \lambda_{c}^{-} \bar{\epsilon}^{\dot{\beta} c}\right) \partial_{\alpha \dot{\beta}} \bar{W}\right]=\frac{1}{8} \hat{\delta}_{\bar{\lambda}_{c}^{+} \epsilon^{\alpha c} \bar{W} .}
\end{aligned}
$$

Hence, the on-shell commutator of the hidden supersymmetry transformations (2.20) with the $R$-symmetry ones (2.21) gives the manifest supersymmetry (2.22), with the bracket parameter $\bar{\lambda}_{c}^{+} \epsilon^{\alpha c}$, in agreement with $\mathcal{N}=4$ supersymmetry algebra.

Let us now evaluate the commutators of the $R$-symmetry transformations (2.21) with the manifest supersymmetry transformations (2.22). We obtain

$$
\begin{aligned}
& \left(\delta_{\lambda} \hat{\delta}_{\epsilon}-\hat{\delta}_{\epsilon} \delta_{\lambda}\right) q_{a}^{+}=-\frac{1}{4}\left(\epsilon^{\alpha i} \lambda_{i a} D_{\alpha}^{+} W+\bar{\epsilon}^{\dot{\alpha} i} \bar{\lambda}_{i a} \bar{D}_{\dot{\alpha}}^{+} \bar{W}\right)=-\delta_{\lambda_{i a} \epsilon^{\alpha i}} q_{a}^{+}, \\
& \left(\delta_{\lambda} \hat{\delta}_{\epsilon}-\hat{\delta}_{\epsilon} \delta_{\lambda}\right) W=-\frac{1}{2} \bar{\epsilon}^{\dot{\alpha} i} \bar{\lambda}_{i}^{a} \bar{D}_{\dot{\alpha}}^{-} q_{a}^{+}=-\delta_{\lambda_{i a} \epsilon^{\alpha i}} W \\
& \left(\delta_{\lambda} \hat{\delta}_{\epsilon}-\hat{\delta}_{\epsilon} \delta_{\lambda}\right) \bar{W}=-\frac{1}{2} \epsilon^{\alpha i} \lambda_{i}^{a} D_{\alpha}^{-} q_{a}^{+}=-\delta_{\lambda_{i a} \epsilon^{\alpha i}} \bar{W} .
\end{aligned}
$$

So the on-shell commutator of the $R$-symmetry transformations (2.21) with the manifest supersymmetry transformations (2.22) yields those of hidden supersymmetry (2.20), with the bracket parameter $\left.\left(\epsilon_{a}^{\alpha}\right)\right)_{\mathrm{br}}=\lambda_{i a} \epsilon^{\alpha i}$.

Finally, we consider the commutator of the $R$-symmetry transformations with itself. We have

$$
\begin{aligned}
\left(\delta_{\lambda_{1}} \delta_{\lambda_{2}}-\delta_{\lambda_{2}} \delta_{\lambda_{1}}\right) q_{a}^{+}= & \frac{1}{8}\left[\lambda_{(\mathrm{PG}) a}^{b} q_{b}^{+}+\frac{1}{2} \lambda \bar{\theta}^{+\dot{\alpha}} \frac{\partial}{\partial \bar{\theta}+\dot{\alpha}} q_{a}^{+}+\frac{1}{2} \bar{\lambda} \theta^{+\alpha} \frac{\partial}{\partial \theta^{+\alpha}} q_{a}^{+}\right] \\
& -\frac{1}{8} \lambda_{j}^{i}\left(u_{i}^{+} \frac{\partial}{\partial u^{+j}}+u_{i}^{-} \frac{\partial}{\partial u^{-j}}\right) q_{a}^{+} \\
\left(\delta_{\lambda_{1}} \delta_{\lambda_{2}}-\delta_{\lambda_{2}} \delta_{\lambda_{1}}\right) \bar{W}= & -\frac{1}{8} \lambda_{j}^{i}\left(u_{i}^{+} \frac{\partial}{\partial u^{+j}}+u_{i}^{-} \frac{\partial}{\partial u^{-j}}\right) \bar{W} \\
& +\frac{1}{8}\left[\frac{1}{2} \lambda \bar{\theta}^{-\dot{\alpha}} \frac{\partial}{\partial \bar{\theta}^{-\dot{\alpha}}} \bar{W}+\frac{1}{2} \lambda \bar{\theta}^{+\dot{\alpha}} \frac{\partial}{\partial \bar{\theta}^{+\dot{\alpha}}} \bar{W}+\frac{1}{2} \bar{\lambda} \theta^{+\alpha} \frac{\partial}{\partial \theta^{+\alpha}} \bar{W}-\lambda \bar{W}\right],
\end{aligned}
$$


where

$$
\lambda^{i j}=\lambda_{2}^{(i a} \bar{\lambda}_{1 a}^{j)}-\lambda_{1}^{(i a} \bar{\lambda}_{2 a}^{j)}, \quad \lambda=\lambda_{2}^{i a} \bar{\lambda}_{1 i a}-\lambda_{1}^{i a} \bar{\lambda}_{2 i a}, \quad \lambda_{(\mathrm{PG})}^{a b}=\lambda_{2}^{i(a} \bar{\lambda}_{1 i}^{b)}-\lambda_{1}^{i(a} \bar{\lambda}_{2 i}^{b)} .
$$

Here, the parameters $\lambda^{i j}$ correspond to $\mathrm{SU}(2)_{R}$ transformations, the parameter $\lambda(\bar{\lambda}=-\lambda)$ corresponds to the additional $\mathrm{U}(1)_{R}$ symmetry and $\lambda_{(\mathrm{PG})}^{a b}$ are associated with the $\mathrm{SU}(2)_{\mathrm{PG}}$ symmetry commuting with both $\mathcal{N}=2$ supersymmetry and $\mathrm{U}(2)_{R}$ symmetry.

Thus the on-shell closure of the implicit $R$-symmetry transformations yields the linear $\mathrm{U}(2)_{R}$ and $\mathrm{SU}(2)_{\mathrm{PG}}$ transformations, once again in agreement with the action of the coset $\mathrm{SU}(4)_{R} /\left[\mathrm{U}(2)_{R} \times \mathrm{SU}(2)_{\mathrm{PG}}\right]$ part of the full automorphism symmetry $\mathrm{SU}(4)_{R}$ on $\mathcal{N}=4$ supersymmetry algebra.

Note that the calculation of the brackets (2.25) is not as straightforward as that of the previous Lie brackets. Some details of it are collected in appendices A and B.

\subsection{Effective action}

The leading low-energy term in the effective action of $\mathcal{N}=2$ SYM theory in $\mathcal{N}=2$ superspace has the form (see, e.g., the reviews $[4,5]$ )

$$
\Gamma_{0}=\int d^{12} z d u \mathcal{H}(W, \bar{W}), \quad \mathcal{H}(W, \bar{W})=c \ln \left(\frac{W}{\Lambda}\right) \ln \left(\frac{\bar{W}}{\Lambda}\right),
$$

where $\Lambda$ is an arbitrary scale. ${ }^{1}$

The complete $\mathcal{N}=4 \mathrm{SYM}$ effective action is an extension of the effective action (2.27) by hypermultiplet-dependent terms. It was first found in [7]:

$$
\Gamma=\int d^{12} z d u\left[c \ln \left(\frac{W}{\Lambda}\right) \ln \left(\frac{\bar{W}}{\Lambda}\right)+\mathcal{L}\left(-2 \frac{q^{+a} q_{a}^{-}}{W \bar{W}}\right)\right]
$$

where

$$
\mathcal{L}(Z)=c \sum_{n=1}^{\infty} \frac{Z^{n}}{n^{2}(n+1)}=c\left[(Z-1) \frac{\ln (1-Z)}{Z}+\operatorname{Li}_{2}(Z)-1\right]
$$

and $\operatorname{Li}_{2}(Z)$ is the Euler dilogarithm function. The part containing $q^{+a}$ is fixed by the requirement that the effective action $\Gamma$ be invariant under both manifest off-shell $\mathcal{N}=2$ supersymmetry and hidden on-shell $\mathcal{N}=2$ supersymmetry. As a result, the effective action (2.28) is an invariant of $\mathcal{N}=4$ supersymmetry and depends on all fields of $\mathcal{N}=4$ gauge multiplet.

Now we will show that the effective action (2.28) can be alternatively derived from (2.27) by imposing the requirement of $R$-symmetry instead of invariance under the hidden $\mathcal{N}=2$ supersymmetry. To this end, let us consider the variation of (2.27) under the transformations (2.21). Based on the reality reasonings, it is enough to concentrate only on that part of the transformations which involves the parameter $\lambda^{i a}$, neglecting the

\footnotetext{
${ }^{1}$ In fact, the action does not depend on $\Lambda$ in virtue of the (anti)chirality of $(\bar{W}) W$.
} 
part containing $\bar{\lambda}^{i a}$,

$$
\begin{aligned}
& \delta \int d^{12} z d u c \ln \left(\frac{W}{\Lambda}\right) \ln \left(\frac{\bar{W}}{\Lambda}\right) \\
& =\frac{c}{2} \int d^{12} z d u \ln \left(\frac{W}{\Lambda}\right) \frac{2 \lambda^{-a} q_{a}^{+}+\lambda^{-a} \theta^{+\alpha} D_{\alpha}^{-} q_{a}^{+}-\lambda^{+a} \theta^{-\alpha} D_{\alpha}^{-} q_{a}^{+}}{\bar{W}} \\
& =\frac{c}{2} \int d^{12} z d u \frac{-\lambda^{-a} \theta^{+\alpha} D_{\alpha}^{-} W q_{a}^{+}+\lambda^{+a} \theta^{-\alpha} D_{\alpha}^{-} W q_{a}^{+}}{W \bar{W}} .
\end{aligned}
$$

Due to the property that $\int d^{12} z d u \frac{q^{+a} \lambda_{a}^{-}}{\bar{W}}=0$, the variation (2.30) can be canceled by adding the new term to $\mathcal{H}$ :

$$
\mathcal{L}_{1}=-c \frac{q^{+a} q_{a}^{-}}{W \bar{W}} .
$$

Evaluating the variation of the sum $\mathcal{H}+\mathcal{L}_{1}$, we obtain

$$
\begin{aligned}
\delta \int d^{12} z d u\left[\mathcal{H}(W, \bar{W})+\mathcal{L}_{1}\right]= & \frac{c}{2} \int d^{12} z d u \frac{q^{+b} q_{b}^{-}}{W \bar{W}^{2}}\left[\lambda^{-a} q_{a}^{+}-\lambda^{+a} q_{a}^{-}\right. \\
& \left.+\left(\lambda^{-a} \theta^{+\alpha}-\lambda^{+a} \theta^{-\alpha}\right) D_{\alpha}^{+} q_{a}^{-}\right] \\
= & \frac{c}{2} \int d^{12} z d u \frac{q^{+b} q_{b}^{-}}{W \bar{W}^{2}}\left[2 \lambda^{-a} q_{a}^{+}+\left(\lambda^{-a} \theta^{+\alpha}-\lambda^{+a} \theta^{-\alpha}\right) D_{\alpha}^{-} q_{a}^{+}\right] .
\end{aligned}
$$

Consider the last term in some detail

$$
\begin{aligned}
\frac{c}{2} \int d^{12} z d u \frac{q^{+b} q_{b}^{-}}{W \bar{W}^{2}}\left(\lambda^{-a} \theta^{+\alpha}-\lambda^{+a} \theta^{-\alpha}\right) D_{\alpha}^{-} q_{a}^{+} \\
=\frac{c}{2} \int d^{12} z d u\left[\frac{-2 \lambda^{-a} W+\left(\lambda^{-a} \theta^{+\alpha}-\lambda^{+a} \theta^{-\alpha}\right) D_{\alpha}^{-} W}{(W \bar{W})^{2}} q_{a}^{+}\left(q^{+b} q_{b}^{-}\right)\right. \\
\left.\quad-\left(\lambda^{-a} \theta^{+\alpha}-\lambda^{+a} \theta^{-\alpha}\right) \frac{q^{+b} D_{\alpha}^{-} q_{b}^{-} q_{a}^{+}}{W \bar{W}^{2}}\right] \\
=\frac{c}{2} \int d^{12} z d u\left[\frac{-2 \lambda^{-a} W+\left(\lambda^{-a} \theta^{+\alpha}-\lambda^{+a} \theta^{-\alpha}\right) D_{\alpha}^{-} W}{(W \bar{W})^{2}} q_{a}^{+}\left(q^{+b} q_{b}^{-}\right)\right. \\
\left.\quad-\left(\lambda^{-a} \theta^{+\alpha}-\lambda^{+a} \theta^{-\alpha}\right) \frac{q^{+b} q_{b}^{-} D_{\alpha}^{-} q_{a}^{+}}{2 W \bar{W}^{2}}\right] .
\end{aligned}
$$

Here we have used various properties of the involved superfields (chirality, analyticity), as well as the integration by parts with respect to the spinor derivative in the second line and cyclic identities for the $\mathrm{SU}(2)$ doublet indices in the third line. Observe that the last term in the third line equals, modulo the minus sign, the variation we started with. Hence,

$$
\begin{aligned}
& \frac{c}{2} \int d^{12} z d u \frac{q^{+b} q_{b}^{-}}{W \bar{W}^{2}}\left(\lambda^{-a} \theta^{+\alpha}-\lambda^{+a} \theta^{-\alpha}\right) D_{\alpha}^{-} q_{a}^{+} \\
& =\frac{c}{3} \int d^{12} z d u\left[\frac{-2 \lambda^{-a} W+\left(\lambda^{-a} \theta^{+\alpha}-\lambda^{+a} \theta^{-\alpha}\right) D_{\alpha}^{-} W}{(W \bar{W})^{2}} q_{a}^{+}\left(q^{+b} q_{b}^{-}\right)\right] .
\end{aligned}
$$


Plugging (2.34) into (2.32), we obtain

$$
\begin{aligned}
& \delta \int d^{12} z d u\left[\mathcal{H}(W, \bar{W})+\mathcal{L}_{1}\right] \\
& =\frac{c}{3} \int d^{12} z d u \frac{q^{+b} q_{b}^{-}}{(W \bar{W})^{2}}\left[\lambda^{-a} q_{a}^{+} W+\left(\lambda^{-a} \theta^{+\alpha}-\lambda^{+a} \theta^{-\alpha}\right) D_{\alpha}^{-} W q_{a}^{+}\right] .
\end{aligned}
$$

This variation is canceled by adding the new proper term to (2.27),

$$
\mathcal{L}_{2}=\frac{c}{3} \frac{\left(q^{+a} q_{a}^{-}\right)^{2}}{(W \bar{W})^{2}}
$$

Continuing the iterative process, one can find that

$$
\mathcal{L}_{n}=\frac{c}{n^{2}(n+1)} \frac{\left(-2 q^{+a} q_{a}^{-}\right)^{n}}{(W \bar{W})^{n}} .
$$

Summing up all $\mathcal{L}_{n}$, one recovers the effective action (2.28).

One can directly verify that $(2.28)$ is invariant under the $R$-symmetry transformations (2.21). Once again, we limit our attention to terms with the parameter $\lambda^{i a}$ :

$$
\begin{aligned}
\delta \Gamma= & c \int d^{12} z d u\left[\frac{\left(\lambda^{+a} \theta^{-\alpha}-\lambda^{-a} \theta^{+\alpha}\right) D_{\alpha}^{-} W q_{a}^{+}}{2 W \bar{W}}\right. \\
& -\mathcal{L}^{\prime}(Z) \frac{q^{+a}\left[\lambda_{a}^{-} W+\left(\lambda_{a}^{-} \theta^{+\alpha}-\lambda_{a}^{+} \theta^{-\alpha}\right) D_{\alpha}^{-} W\right]}{W \bar{W}} \\
& \left.-Z \mathcal{L}^{\prime}(Z) \frac{\left[2 \lambda^{-a} q_{a}^{+}+\left(\lambda^{-a} \theta^{+\alpha}-\lambda^{+a} \theta^{-\alpha}\right) D_{\alpha}^{-} q_{a}^{+}\right]}{2 \bar{W}}\right] .
\end{aligned}
$$

This expression can be simplified, using the identity

$$
\begin{aligned}
& \int d^{12} z d u \frac{Z \mathcal{L}^{\prime}(Z)}{\bar{W}}\left[2 \lambda^{-a} q_{a}^{+}+\left(\lambda^{-a} \theta^{+\alpha}-\lambda^{+a} \theta^{-\alpha}\right) D_{\alpha}^{-} q_{a}^{+}\right] \\
& =\int d^{12} z d u\left[2 \mathcal{L}^{\prime}(Z)-1\right]\left[\frac{\lambda^{-a} q_{a}^{+}}{\bar{W}}+\left(\lambda^{-a} \theta^{+\alpha}-\lambda^{+a} \theta^{-\alpha}\right) \frac{q_{a}^{+} D_{\alpha}^{-} W}{W \bar{W}}\right],
\end{aligned}
$$

which is deduced by integrating by parts with respect to the spinor derivative and applying to the definition of the function $\mathcal{L}(Z)(2.28)$.

Thus we obtain

$$
\begin{aligned}
\delta \Gamma= & c \int d^{12} z d u\left[\frac{\left(\lambda^{+a} \theta^{-\alpha}-\lambda^{-a} \theta^{+\alpha}\right) D_{\alpha}^{-} W q_{a}^{+}}{2 W \bar{W}}\right. \\
& \left.-\mathcal{L}^{\prime}(Z) \frac{q^{+a}\left[\lambda_{a}^{-} W+\left(\lambda_{a}^{-} \theta^{+\alpha}-\lambda_{a}^{+} \theta^{-\alpha}\right) D_{\alpha}^{-} W\right]}{W \bar{W}}\right] \\
& -\frac{1}{2} \int d^{12} z d u\left[2 \mathcal{L}^{\prime}(Z)-1\right]\left[\frac{\lambda^{-a} q_{a}^{+}}{\bar{W}}+\left(\lambda^{-a} \theta^{+\alpha}-\lambda^{+a} \theta^{-\alpha}\right) \frac{q_{a}^{+} D_{\alpha}^{-} W}{W \bar{W}}\right]=0 .
\end{aligned}
$$

To summarize, the requirement of invariance under the $R$-symmetry transformations allowed us to completely restore the hypermultiplet dependence of $\mathcal{N}=4$ supersymmetric effective action. 


\subsection{Superconformal invariance of the effective action}

The effective action (2.28) is evidently scale-invariant. In this section we prove that it is actually invariant under the total $4 D, \mathcal{N}=4$ superconformal group $\mathrm{SU}(2,2 \mid 4)$.

Due to the structure of $4 D, \mathcal{N}=4$ superconformal algebra and the $R$-symmetry invariance of the effective action it suffices to show only its $4 D, \mathcal{N}=2$ superconformal invariance. Moreover, it is enough to check just invariance under conformal boosts.

Indeed, the commutator of conformal boosts with the manifest $\mathcal{N}=2$ Poincaré supersymmetry yields the special conformal $\mathcal{N}=2$ supersymmetry. The $\mathcal{N}=4$ completion of the latter is contained in the commutator of conformal boosts with the hidden $\mathcal{N}=2$ supersymmetry which, as we saw, is obtained by commuting the hidden $R$-symmetry with $\mathcal{N}=2$ Poincaré supersymmetry.

To prove the superconformal invariance we should use the transformation rules of the harmonic superspace coordinate, as well as those of the harmonic and spinor derivatives. The harmonic superspace coordinate transformations under conformal boosts in the analytic basis read [3]

$$
\begin{array}{ll}
\delta x^{\alpha \dot{\alpha}}=x^{\beta \dot{\alpha}} k_{\beta \dot{\beta}} x^{\alpha \dot{\beta}}, & \\
\delta \theta^{+\alpha}=\theta^{+\beta} k_{\beta \dot{\beta}} x^{\alpha \dot{\beta}}, & \delta \theta^{-\alpha}=\theta^{-\beta} k_{\beta \dot{\beta}} x^{\alpha \dot{\beta}}-2 i\left(\theta^{-}\right)^{2} \bar{\theta}_{\dot{\beta}}^{+} k^{\alpha \dot{\beta}}, \\
\delta \bar{\theta}^{+\dot{\alpha}}=\bar{\theta}^{+\dot{\beta}} k_{\beta \dot{\beta}} x^{\beta \dot{\alpha}}, & \delta \bar{\theta}^{-\dot{\alpha}}=\bar{\theta}^{-\dot{\beta}} k_{\beta \dot{\beta}} x^{\beta \dot{\alpha}}-2 i\left(\bar{\theta}^{-}\right)^{2} \theta_{\beta}^{+} k^{\beta \dot{\alpha}},
\end{array}
$$

where $k^{\alpha \dot{\alpha}}$ is the corresponding 4 -vector parameter. The transformation law of the harmonics is [3]

$$
\begin{aligned}
\delta u^{+k} & =\Lambda^{++} u_{k}^{-}, \quad \delta u_{k}^{-}=0, \quad \Lambda^{++}=4 i \theta^{+\alpha} k_{\alpha \dot{\alpha}} \bar{\theta}^{+\dot{\alpha}}, \\
D^{--} \Lambda^{++} & =4 i \theta^{-\alpha} k_{\alpha \dot{\alpha}} \bar{\theta}^{+\dot{\alpha}}+4 i \theta^{+\alpha} k_{\alpha \dot{\alpha}} \bar{\theta}^{-\dot{\alpha}} .
\end{aligned}
$$

Next, let us write the superconformal transformations of the harmonic and spinor derivatives

$$
\delta D^{++}=-\Lambda^{++} D^{0}, \quad \delta D^{--}=-\left(D^{--} \Lambda^{++}\right) D^{--}, \quad \delta D_{\alpha}^{+}=-D_{\alpha}^{+}\left(\delta \theta^{-\beta}\right) D_{\beta}^{+} .
$$

Using these transformation rules it is easy to establish the transformation of the superspace integration measure $d Z=d^{12} z d u=d^{4} x d^{4} \theta^{+} d^{4} \theta^{-} d u$,

$$
\begin{aligned}
\delta d Z & =\left(\partial_{\alpha \dot{\alpha}} x^{\alpha \dot{\alpha}}+\partial^{--} \Lambda^{++}-\partial_{+\alpha} \theta^{+\alpha}-\partial_{-\alpha} \theta^{-\alpha}-\bar{\partial}_{+\dot{\alpha}} \bar{\theta}^{+\dot{\alpha}}-\bar{\partial}_{+\dot{\alpha}} \bar{\theta}^{+\alpha}\right) d Z=\Lambda d Z, \\
\Lambda & =4 i\left(\theta_{\alpha}^{-} \bar{\theta}_{\dot{\alpha}}^{+}-\bar{\theta}_{\dot{\alpha}}^{-} \theta_{\alpha}^{+}\right) k^{\alpha \dot{\alpha}} .
\end{aligned}
$$

Let us now consider the transformations of the gauge potentials $V^{ \pm \pm}$. Taking into account the relations (2.41), (2.42), (2.43) one deduces

$$
\delta V^{++}=0, \quad \delta V^{--}=-\left(D^{--} \Lambda^{++}\right) V^{--} .
$$

Using the transformation rules (2.43) and (2.45), it is easy to obtain the transformation law of the superfield strengths $W, \bar{W}$

$$
\delta W=-k_{\alpha \dot{\alpha}}\left(x^{\alpha \dot{\alpha}}+4 i \theta^{-\alpha} \bar{\theta}^{+\dot{\alpha}}\right) W, \quad \delta \bar{W}=-k_{\alpha \dot{\alpha}}\left(x^{\alpha \dot{\alpha}}+4 i \theta^{+\alpha} \bar{\theta}^{-\dot{\alpha}}\right) \bar{W} .
$$


The transformation of the hypermultiplet $q^{ \pm a}$ under the conformal boosts reads [3]

$$
\delta q^{+a}=-k_{\alpha \dot{\alpha}} x^{\alpha \dot{\alpha}} q^{+a}, \quad \delta q^{-a}=\delta\left(D^{--} q^{+a}\right)=-k_{\alpha \dot{\alpha}} x^{\alpha \dot{\alpha}} q^{-a}-4 i\left(\theta_{\alpha}^{-} \bar{\theta}_{\dot{\alpha}}^{+}-\bar{\theta}_{\dot{\alpha}}^{-} \theta_{\alpha}^{+}\right) k^{\alpha \dot{\alpha}} q^{-a}
$$

Now we are prepared to show the conformal invariance of the effective action (2.28).

Let us first show invariance of the logarithmic term

$$
\begin{aligned}
\delta \int & d^{12} z d u c \ln \left(\frac{W}{\Lambda}\right) \ln \left(\frac{\bar{W}}{\Lambda}\right) \\
= & c \int d^{12} z d u\left[4 i\left(\theta_{\alpha}^{-} \bar{\theta}_{\dot{\alpha}}^{+}-\bar{\theta}_{\dot{\alpha}}^{-} \theta_{\alpha}^{+}\right) k^{\alpha \dot{\alpha}} \ln \left(\frac{W}{\Lambda}\right) \ln \left(\frac{\bar{W}}{\Lambda}\right)\right. \\
& \left.-k_{\alpha \dot{\alpha}}\left(x^{\alpha \dot{\alpha}}+4 i \theta^{-\alpha} \bar{\theta}^{+\dot{\alpha}}\right) \ln \left(\frac{\bar{W}}{\Lambda}\right)-k_{\alpha \dot{\alpha}}\left(x^{\alpha \dot{\alpha}}+4 i \theta^{+\alpha} \bar{\theta}^{-\dot{\alpha}}\right) \ln \left(\frac{W}{\Lambda}\right)\right] \\
= & c \int d^{12} z d u\left[-4 i\left(\theta_{\alpha}^{+} \bar{\theta}_{\dot{\alpha}}^{-}+\bar{\theta}_{\dot{\alpha}}^{-} \theta_{\alpha}^{+}\right) k^{\alpha \dot{\alpha}} \ln \left(\frac{W}{\Lambda}\right) \ln \left(\frac{\bar{W}}{\Lambda}\right)\right]=0
\end{aligned}
$$

where we made use of some properties of $W$ and $\bar{W}$, eqs. (2.18), (2.19). Then we check invariance of the generic term in the power expansion of the function $\mathcal{L}(z)$

$$
\begin{aligned}
\delta \int d^{12} z d u\left(\frac{q^{+a} q_{a}^{-}}{W \bar{W}}\right)^{n}= & n \int d^{12} z d u\left(\frac{q^{+a} q_{a}^{-}}{W \bar{W}}\right)^{n}\left[\frac{4 i}{n}\left(\theta_{\alpha}^{-} \bar{\theta}_{\dot{\alpha}}^{+}-\bar{\theta}_{\dot{\alpha}}^{-} \theta_{\alpha}^{+}\right) k^{\alpha \dot{\alpha}}\right. \\
& -k_{\alpha \dot{\alpha}} x^{\alpha \dot{\alpha}}-k_{\alpha \dot{\alpha}} x^{\alpha \dot{\alpha}}-4 i\left(\theta_{\alpha}^{-} \bar{\theta}_{\dot{\alpha}}^{+}-\bar{\theta}_{\dot{\alpha}}^{-} \theta_{\alpha}^{+}\right) k^{\alpha \dot{\alpha}} \\
& \left.+k_{\alpha \dot{\alpha}}\left(x^{\alpha \dot{\alpha}}+4 i \theta^{-\alpha} \bar{\theta}^{+\dot{\alpha}}\right)+k_{\alpha \dot{\alpha}}\left(x^{\alpha \dot{\alpha}}+4 i \theta^{+\alpha} \bar{\theta}^{-\dot{\alpha}}\right)\right]=0 .
\end{aligned}
$$

Here we used, once again, the conditions (2.18) and (2.19), as well as the equations of motion (2.16).

So we have proved that the effective action (2.28) is superconformally invariant on shell. Note that the original "microscopic" action is invariant under the conformal boosts and hence under the whole $\mathcal{N}=4$ superconformal group off shell, without any use of the equations of motion. The latter, like in the case of hidden $\mathcal{N}=4$ supersymmetry and $R$ symmetry, are of need only when checking the correct closure of all these transformations. On the other hand, the effective action reveals the invariance under the hidden $\mathcal{N}=4$ supersymmetry and $R$-symmetry only on shell, so it is quite natural that the same is also valid for $\mathcal{N}=4$ superconformal symmetry.

\section{$35 D, \mathcal{N}=2$ SYM theory}

In this section, we introduce the $R$-symmetry transformations for $5 D, \mathcal{N}=2 \mathrm{SYM}$ theory with the gauge group $\mathrm{SU}(2)$ and construct the complete low-energy effective action by requiring invariance under these $R$-symmetry transformations. We use the notations and conventions of refs. $[3,11]$. The relevant harmonic superspace formalism to large extent is similar to its $4 D, \mathcal{N}=2$ prototype. 


\subsection{Classical action}

The $\mathcal{N}=2$ gauge multiplet in $5 D, \mathcal{N}=1$ harmonic superspace consists of $\mathcal{N}=1$ gauge multiplet represented by the analytic superfield $V^{++}$and the hypermultiplet $q^{+a}$. The $\mathcal{N}=1$ gauge multiplet classical action reads [10]

$$
S_{\mathrm{SYM}}^{\mathcal{N}=1}=\frac{1}{2 g^{2}} \sum_{n=2}^{\infty} \operatorname{tr} \frac{(-i)^{n}}{n} \int d^{13} z d u_{1} \ldots d u_{n} \frac{V^{++}\left(z, u_{1}\right) \ldots V^{++}\left(z, u_{n}\right)}{\left(u_{1}^{+} u_{2}^{+}\right) \ldots\left(u_{n}^{+} u_{1}^{+}\right)},
$$

where $g$ is a coupling constant of mass-dimension $-1 / 2$. The superfield strength is defined in the analytic $\lambda$-frame as

$$
W=\frac{i}{8}\left(D^{+}\right)^{2} V^{--},
$$

where $\left(D^{+}\right)^{2}=D^{+\hat{\alpha}} D_{\hat{\alpha}}^{+}=\Omega^{\hat{\alpha} \hat{\beta}} D_{\hat{\beta}}^{+} D_{\hat{\alpha}}^{+}, \Omega^{\hat{\alpha} \hat{\beta}}$ is the USp(4) invariant skew-symmetric constant "metric" and $V^{--}$is a non-analytic gauge potential related to $V^{++}$by the harmonic flatness condition

$$
D^{++} V^{--}-D^{--} V^{++}+i\left[V^{++}, V^{--}\right]=0 .
$$

The classical action of the hypermultiplet $q^{+a}=\left(q^{+},-\tilde{q}^{+}\right)$in the adjoint representation of the gauge group is written as [2]

$$
S_{q}=\frac{1}{2 g^{2}} \operatorname{tr} \int d \zeta^{-4} q_{a}^{+} \nabla^{++} q^{+a}=\frac{1}{2 g^{2}} \operatorname{tr} \int d \zeta^{-4} q_{a}^{+}\left(D^{++} q^{+a}+i\left[V^{++}, q^{+a}\right]\right),
$$

where $d \zeta^{-4}$ is the analytic superspace integration measure. In addition, this action is invariant under $\mathrm{SU}(2)_{\mathrm{PG}}$ symmetry which transforms $q^{+a}$ as a doublet.

The action of $\mathcal{N}=2$ gauge multiplet in $5 D, \mathcal{N}=1$ harmonic superspace is just the sum of (3.1) and (3.4),

$$
S^{\mathcal{N}=2}=S_{\mathrm{SYM}}^{\mathcal{N}=1}+S_{q}
$$

The action is invariant under the implicit $\mathcal{N}=1$ supersymmetry completing the manifest $\mathcal{N}=1$ supersymmetry to the total $\mathcal{N}=2$ supersymmetry

$$
\delta q_{a}^{+}=-\frac{1}{2}\left(D^{+}\right)^{4}\left[\epsilon_{a \hat{\alpha}} \theta^{-\hat{\alpha}} V^{--}\right], \quad \delta V^{++}=\epsilon_{\hat{\alpha}}^{a} \theta^{+\hat{\alpha}} q_{a}^{+},
$$

where $\epsilon_{a \hat{\alpha}}^{a}$ is the relevant anticommuting parameter and

$$
\left(D^{+}\right)^{4}=-\frac{1}{32}\left(D^{+}\right)^{2}\left(D^{+}\right)^{2} .
$$

\section{$3.2 \quad R$-symmetry transformations}

We define the $R$-symmetry transformations in $5 D, \mathcal{N}=1$ harmonic superspace as

$$
\delta q^{+a}=-\frac{i}{4}\left(D^{+}\right)^{4}\left[\lambda^{+a}\left(\theta^{-}\right)^{2} V^{--}-2 \lambda^{-a} \theta^{+\hat{\alpha}} \theta_{\hat{\alpha}}^{-} V^{--}\right], \quad \delta V^{++}=-i \lambda^{-a}\left(\theta^{+}\right)^{2} q_{a}^{+},
$$

where $\lambda^{i a}$ is the relevant commuting parameter $\left(\overline{\lambda^{i a}}=\lambda_{i a}, \lambda^{+a}=\lambda^{i a} u_{i}^{+}\right)$. These transformations extend the $R$-symmetry group from $\mathrm{SU}(2)_{R} \times \mathrm{SU}(2)_{\mathrm{PG}}$ to $\mathrm{SO}(5)$. The direct check shows that the action (3.5) is invariant off shell under the transformations (3.8). 
Further in this section we consider the case of abelian gauge group. The action (3.5) yields the equations of motion

$$
\left(D^{+}\right)^{2} W=0, \quad D^{++} q_{a}^{+}=0 .
$$

In addition, the superfield strength $W$ satisfies the off-shell constraints

$$
D^{++} W=0, \quad D^{--} W=0,
$$

which follow from the harmonic flatness condition (3.3) and the analyticity of $V^{++}$.

On the shell of the equations of motion (3.9) the transformations of the hidden supersymmetry (3.6) are reduced to

$$
\delta q_{a}^{ \pm}=\frac{i}{2} \epsilon_{a}^{\hat{\alpha}}\left(D_{\hat{\alpha}}^{ \pm} W\right), \quad \delta W=-\frac{i}{8} \epsilon_{\hat{\alpha}}^{a} D^{-\hat{\alpha}} q_{a}^{+}+\frac{i}{8} \epsilon_{\hat{\alpha}}^{a} D^{+\hat{\alpha}} q_{a}^{-},
$$

where $q^{-a}=D^{--} q^{+a}$. The $R$-symmetry transformations (3.8) take the form

$$
\begin{aligned}
& \delta q_{a}^{+}=-\frac{1}{2}\left(\lambda_{a}^{+} W-\lambda_{a}^{+} \theta^{-\hat{\alpha}} D_{\hat{\alpha}}^{+} W+\lambda_{a}^{-} \theta^{+\hat{\alpha}} D_{\hat{\alpha}}^{+} W\right), \\
& \delta W=\frac{1}{4}\left(2 \lambda^{+a} q_{a}^{-}-2 \lambda^{-a} q_{a}^{+}+\lambda^{-a} \theta^{+\hat{\alpha}} D_{\hat{\alpha}}^{+} q_{a}^{-}-\lambda^{+a} \theta^{-\hat{\alpha}} D_{\hat{\alpha}}^{+} q_{a}^{-}\right) .
\end{aligned}
$$

Now we can consider the commutator of supersymmetry transformations with those of $R$-symmetry. The variation of general superfield under the manifest supersymmetry reads

$$
\hat{\delta} \Phi=-\epsilon^{+\hat{\alpha}} \frac{\partial \Phi}{\partial \theta^{+\hat{\alpha}}}-\epsilon^{\hat{-} \hat{\alpha}} \frac{\partial \Phi}{\partial \theta^{-\hat{\alpha}}}-2 i \epsilon^{-\hat{\alpha}} \theta^{+\hat{\beta}} \partial_{\hat{\alpha} \hat{\beta}} \Phi,
$$

where $\epsilon_{\hat{\alpha}}^{ \pm}=\epsilon_{\hat{\alpha}}^{a} u_{a}^{ \pm}$are the relevant anticommuting parameters.

Let us first consider the commutators of the hidden supersymmetry transformations (3.11) with the $R$-symmetry transformations (3.12). One can show that

$$
\begin{aligned}
\left(\delta_{\lambda} \delta_{\epsilon}-\delta_{\epsilon} \delta_{\lambda}\right) q^{+a} & =\frac{i}{8}\left(-\lambda_{c}^{-} \epsilon^{\hat{\alpha} c} \frac{\partial q^{+a}}{\partial \theta^{-\hat{\alpha}}}-2 i \lambda_{c}^{-} \epsilon^{\hat{\alpha} c} \theta^{+\hat{\beta}} \partial_{\hat{\alpha} \hat{\beta}} q^{+a}\right)=\frac{i}{8} \hat{\delta}_{\lambda_{c}^{i} \epsilon^{\hat{\alpha} c} q^{+a}} \\
\left(\delta_{\lambda} \delta_{\epsilon}-\delta_{\epsilon} \delta_{\lambda}\right) W & =\frac{i}{8}\left(-\lambda_{c}^{+} \epsilon^{\hat{\alpha} c} \frac{\partial W}{\partial \theta^{+\hat{\alpha}}}-\lambda_{c}^{-} \epsilon^{\hat{\alpha} c} \frac{\partial W}{\partial \theta^{-\hat{\alpha}}}-2 i \lambda_{c}^{-} \epsilon^{\hat{\alpha} c} \theta^{+\hat{\beta}} \partial_{\hat{\alpha} \hat{\beta}} W\right)=\frac{i}{8} \hat{\delta}_{\lambda_{c}^{i} \epsilon^{\hat{\alpha} c} W}
\end{aligned}
$$

Hence, the on-shell commutator of the hidden supersymmetry transformations with the $R$ symmetry transformations gives the manifest supersymmetry with the bracket parameter $\lambda_{c}^{i} \epsilon^{\hat{\alpha} c}$, as expected.

Let us now consider the commutators of the $R$-symmetry transformations (3.12) with the manifest supersymmetry transformations (3.17). They are given by

$$
\begin{aligned}
\left(\delta_{\lambda} \hat{\delta}_{\epsilon}-\hat{\delta}_{\epsilon} \delta_{\lambda}\right) q^{+a} & =-\frac{1}{2} \epsilon_{i}^{\hat{\alpha}} \lambda^{i a} D_{\hat{\alpha}}^{+} W=i \hat{\delta}_{\lambda^{i a} \epsilon_{i}^{\hat{\alpha}}} q^{+a} \\
\left(\delta_{\lambda} \hat{\delta}_{\epsilon}-\hat{\delta}_{\epsilon} \delta_{\lambda}\right) W & =-\frac{1}{4} \epsilon_{i}^{\hat{\alpha}} \lambda^{i a} D_{\hat{\alpha}}^{+} q_{a}^{-}=i \hat{\delta}_{\lambda^{i a} \epsilon_{i}^{\hat{\alpha}}} W .
\end{aligned}
$$


Thus the on-shell commutator of $R$-symmetry with the manifest supersymmetry yields the hidden supersymmetry with the bracket parameter $\lambda^{i a} \epsilon_{i}^{\hat{\alpha}}$.

At last, one can consider the commutator of $R$-symmetry transformations (3.12) with themselves:

$$
\begin{aligned}
& \left(\delta_{\lambda_{1}} \delta_{\lambda_{2}}-\delta_{\lambda_{2}} \delta_{\lambda_{1}}\right) q_{a}^{+}=\frac{1}{8}\left(\lambda_{2 a}^{i} \lambda_{1 i}^{b}-\lambda_{1 a}^{i} \lambda_{2 i}^{b}\right) q_{b}^{+}-\frac{1}{8}\left(\lambda_{2}^{i a} \lambda_{1 a j}-\lambda_{1}^{i a} \lambda_{2 a j}\right)\left(u_{i}^{+} \frac{\partial}{\partial u_{j}^{+}}+u_{i}^{-} \frac{\partial}{\partial u_{j}^{-}}\right) q_{a}^{+}, \\
& \left(\delta_{\lambda_{1}} \delta_{\lambda_{2}}-\delta_{\lambda_{2}} \delta_{\lambda_{1}}\right) W=-\frac{1}{8}\left(\lambda_{2}^{i a} \lambda_{1 j a}-\lambda_{1}^{i a} \lambda_{2 j a}\right)\left(u_{i}^{+} \frac{\partial}{\partial u_{j}^{+}}+u_{i}^{-} \frac{\partial}{\partial u_{j}^{-}}\right) W .
\end{aligned}
$$

The details of the derivation are given in appendices A and B. We observe that the on-shell commutator of two hidden $R$-symmetry transformations yields manifest linear $\mathrm{SU}(2)_{R}$ and $\mathrm{SU}(2)_{\mathrm{PG}}$ transformations, as should be.

\subsection{Effective action}

The part of the superfield $\mathcal{N}=1$ SYM effective action containing the component fourderivative term of the gauge field reads [11]

$$
S_{0}=c_{0} \int d^{13} z d u W \ln \frac{W}{\Lambda},
$$

where $W$ is the abelian gauge superfield strength, $\Lambda$ is a scale parameter and $c_{0}$ is a dimensionless constant.

The variation of action (3.17) under the transformation (3.12) is as follows

$$
\begin{aligned}
\delta S_{0} & =c_{0} \int d^{13} z d u \ln W \delta W \\
& =\frac{c_{0}}{4} \int d^{13} z d u \ln W\left(2 \lambda^{+a} q_{a}^{-}-2 \lambda^{-a} q_{a}^{+}+\lambda^{-a} \theta^{+\hat{\alpha}} D_{\hat{\alpha}}^{+} q_{a}^{-}-\lambda^{+a} \theta^{-\hat{\alpha}} D_{\hat{\alpha}}^{+} q_{a}^{-}\right) \\
& =-\frac{c_{0}}{4} \int d^{13} z d u \frac{\lambda^{-a} \theta^{+\hat{\alpha}} D_{\hat{\alpha}}^{+} W q_{a}^{-}-\lambda^{+a} \theta^{-\hat{\alpha}} D_{\hat{\alpha}}^{+} W q_{a}^{-}}{W} .
\end{aligned}
$$

It can be partially canceled by variation of the extra term

$$
S_{1}=c_{1} \int d^{13} z d u \frac{q^{+a} q_{a}^{-}}{W} .
$$

The variation of (3.19) reads

$$
\begin{aligned}
\delta S_{1}= & c_{1} \delta \int d^{13} z d u \frac{q^{+a} q_{a}^{-}}{W} \\
= & -c_{1} \int d^{13} z d u \frac{\left(\lambda^{+a} W-\lambda^{+a} \theta^{-\hat{\alpha}} D_{\hat{\alpha}}^{+} W+\lambda^{-a} \theta^{+\hat{\alpha}} D_{\hat{\alpha}}^{+} W\right) q_{a}^{-}}{W} \\
& -\frac{c_{1}}{4} \int d^{13} z d u \frac{q^{+b} q_{b}^{-}}{W^{2}}\left(2 \lambda^{+a} q_{a}^{-}-2 \lambda^{-a} q_{a}^{+}+\lambda^{-a} \theta^{+\hat{\alpha}} D_{\hat{\alpha}}^{+} q_{a}^{-}-\lambda^{+a} \theta^{-\hat{\alpha}} D_{\hat{\alpha}}^{+} q_{a}^{-}\right) .
\end{aligned}
$$

Due to the property $\int d^{13} z d u \lambda^{+a} q_{a}^{-}=0$, the first term in (3.20) exactly cancels (3.18), provided that $c_{1}=-c_{0} / 4$. 
Hence,

$$
\begin{aligned}
\delta\left(S_{0}+S_{1}\right) & =-\frac{c_{1}}{4} \int d^{13} z d u \frac{q^{+b} q_{b}^{-}}{W^{2}}\left[2 \lambda^{+a} q_{a}^{-}-2 \lambda^{-a} q_{a}^{+}+\left(\lambda^{-a} \theta^{+\hat{\alpha}}-\lambda^{+a} \theta^{-\hat{\alpha}}\right) D_{\hat{\alpha}}^{+} q_{a}^{-}\right] \\
& =-\frac{c_{1}}{4} \int d^{13} z d u \frac{q^{+b} q_{b}^{-}}{W^{2}}\left[4 \lambda^{+a} q_{a}^{-}+\left(\lambda^{-a} \theta^{+\hat{\alpha}}-\lambda^{+a} \theta^{-\hat{\alpha}}\right) D_{\hat{\alpha}}^{+} q_{a}^{-}\right] .
\end{aligned}
$$

Consider the last term here in more detail:

$$
\begin{gathered}
-\frac{c_{1}}{4} \int d^{13} z d u \frac{q^{+b} q_{b}^{-}}{W^{2}}\left(\lambda^{-a} \theta^{+\hat{\alpha}}-\lambda^{+a} \theta^{-\hat{\alpha}}\right) D_{\hat{\alpha}}^{+} q_{a}^{-} \\
=-\frac{c_{1}}{4} \int d^{13} z d u\left[\frac{-4 \lambda^{+a} W+2\left(\lambda^{-a} \theta^{+\hat{\alpha}}-\lambda^{+a} \theta^{-\hat{\alpha}}\right) D_{\hat{\alpha}}^{+} W}{W^{3}} q_{a}^{-}\left(q^{+b} q_{b}^{-}\right)\right. \\
\left.-\left(\lambda^{-a} \theta^{+\hat{\alpha}}-\lambda^{+a} \theta^{-\hat{\alpha}}\right) \frac{q^{+b} D_{\hat{\alpha}}^{+} q_{b}^{-} q_{a}^{-}}{W^{2}}\right] \\
=-\frac{c_{1}}{4} \int d^{13} z d u\left[\frac{-4 \lambda^{+a} W+2\left(\lambda^{-a} \theta^{+\hat{\alpha}}-\lambda^{+a} \theta^{-\hat{\alpha}}\right) D_{\hat{\alpha}}^{+} W}{W^{3}} q_{a}^{-}\left(q^{+b} q_{b}^{-}\right)\right. \\
\left.-\left(\lambda^{-a} \theta^{+\hat{\alpha}}-\lambda^{+a} \theta^{-\hat{\alpha}}\right) \frac{q^{+b} q_{b}^{-} D_{\hat{\alpha}}^{+} q_{a}^{-}}{2 W^{2}}\right] .
\end{gathered}
$$

We used the integration by parts with respect to the spinor derivative in the second line and cyclic identities for $\mathrm{SU}(2)$ indices in the third line. We observe that the last term in the third line equals the expression we started with, but with he minus sign. Hence,

$$
\begin{aligned}
& -\frac{c_{1}}{4} \int d^{13} z d u \frac{q^{+b} q_{b}^{-}}{W^{2}}\left(\lambda^{-a} \theta^{+\hat{\alpha}}-\lambda^{+a} \theta^{-\hat{\alpha}}\right) D_{\hat{\alpha}}^{+} q_{a}^{-} \\
& =-\frac{c_{1}}{3} \int d^{13} z d u \frac{\left[-2 \lambda^{+a} W+\left(\lambda^{-a} \theta^{+\hat{\alpha}}-\lambda^{+a} \theta^{-\hat{\alpha}}\right) D_{\hat{\alpha}}^{+} W\right] q_{a}^{-}\left(q^{+b} q_{b}^{-}\right)}{W^{3}} .
\end{aligned}
$$

Substituting (3.23) into (3.21), we obtain

$$
\delta\left(S_{0}+S_{1}\right)=-\frac{c_{1}}{3} \int d^{13} z d u \frac{\left(\lambda_{a}^{+} W-\lambda_{a}^{+} \theta^{-\hat{\alpha}} D_{\hat{\alpha}}^{+} W+\lambda_{a}^{-} \theta^{+\hat{\alpha}} D_{\hat{\alpha}}^{+} W\right) q_{a}^{-}\left(q^{+b} q_{b}^{-}\right)}{W^{3}} .
$$

Once again, the variation of (3.24) can be partially canceled by the variation of the additional term

$$
S_{2}=c_{2} \int d^{13} z d u \frac{\left(q^{+a} q_{a}^{-}\right)^{2}}{W^{3}}, \quad c_{2}=-\frac{c_{1}}{6} .
$$

Finally, we consider the general expression

$$
S=\int d^{13} z d u\left[W \ln \frac{W}{\Lambda}+\sum_{n=1}^{\infty} c_{n} \frac{\left(q^{+a} q_{a}^{-}\right)^{n}}{W^{2 n-1}}\right] .
$$

One can show that this expression is invariant under the transformation (3.12), if

$$
-(n+1) c_{n+1}=\frac{n(2 n-1)}{n+2} c_{n}
$$


Therefore, the action (3.26) is equal to

$$
S=c_{0} \int d^{13} z d u W\left[\ln \frac{W}{\Lambda}+\frac{1}{2} H(Z)\right]
$$

where

$$
Z=\frac{q^{+a} q_{a}^{-}}{W^{2}}
$$

and

$$
H(Z)=1+2 \ln \frac{1+\sqrt{1+2 Z}}{2}+\frac{2}{3} \frac{1}{1+\sqrt{1+2 Z}}-\frac{4}{3} \sqrt{1+2 Z} .
$$

This expression coincides with the one obtained in [9] by resorting to hidden supersymmetry instead of $R$-symmetry. ${ }^{2}$

One can also directly verify that (3.28) in invariant under the $R$-symmetry transformations

$$
\begin{aligned}
\delta S= & c_{0} \int d^{13} z d u\left[\frac{\left(\lambda^{+a} \theta^{-\hat{\alpha}}-\lambda^{-a} \theta^{+\hat{\alpha}}\right) D_{\hat{\alpha}}^{+} W q_{a}^{-}}{4 W}\right. \\
& -\frac{H^{\prime}(Z)\left[\lambda^{+a} W-\left(\lambda^{+a} \theta^{-\hat{\alpha}}-\lambda^{-a} \theta^{+\hat{\alpha}}\right) D_{\hat{\alpha}}^{+} W\right] q_{a}^{-}}{2 W} \\
& \left.+\frac{1}{8}\left[H(Z)-2 Z H^{\prime}(Z)\right]\left[4 \lambda^{+a} q_{a}^{-}+\left(\lambda^{-a} \theta^{+\hat{\alpha}}-\lambda^{+a} \theta^{-\hat{\alpha}}\right) D_{\hat{\alpha}}^{+} q_{a}^{-}\right]\right] .
\end{aligned}
$$

This expression can be simplified with the help of the identity

$$
\begin{aligned}
& \int d z^{13} d u\left[H(Z)-2 Z H^{\prime}(Z)\right]\left[4 \lambda^{+a} q_{a}^{-}+\left(\lambda^{-a} \theta^{+\hat{\alpha}}-\lambda^{+a} \theta^{-\hat{\alpha}}\right) D_{\hat{\alpha}}^{+} q_{a}^{-}\right] \\
& =\int d z^{13} d u 2\left[1+2 H^{\prime}(Z)\right]\left[\left(\lambda^{-a} \theta^{+\hat{\alpha}}-\lambda^{+a} \theta^{-\hat{\alpha}}\right) \frac{q_{a}^{-} D_{\hat{\alpha}}^{+} W}{W}+\lambda^{+a} q_{a}^{-}\right]
\end{aligned}
$$

which is derived by employing the integration by parts with respect to the spinor derivative and using the definition of the function $H(Z)$ (3.30).

Thus we obtain

$$
\begin{aligned}
\delta S= & c_{0} \int d^{13} z d u\left[\frac{\left(\lambda^{+a} \theta^{-\hat{\alpha}}-\lambda^{-a} \theta^{+\hat{\alpha}}\right) D_{\hat{\alpha}}^{+} W q_{a}^{-}}{4 W}\right. \\
& \left.-\frac{H^{\prime}(Z)\left[\lambda^{+a} W-\left(\lambda^{+a} \theta^{-\hat{\alpha}}-\lambda^{-a} \theta^{+\hat{\alpha}}\right) D_{\hat{\alpha}}^{+} W\right] q_{a}^{-}}{2 W}\right] \\
& +c_{0} \int d z^{13} d u \frac{2}{8}\left[1+2 H^{\prime}(Z)\right]\left[\left(\lambda^{-a} \theta^{+\hat{\alpha}}-\lambda^{+a} \theta^{-\hat{\alpha}}\right) \frac{q_{a}^{-} D_{\hat{\alpha}}^{+} W}{W}+\lambda^{+a} q_{a}^{-}\right]=0 .
\end{aligned}
$$

We conclude that the condition of invariance under $R$-symmetry can be employed instead of the demand of hidden supersymmetry in order to construct the complete $5 D, \mathcal{N}=$ 2 invariant superspace functional, starting from the functional which is invariant under the manifest $\mathcal{N}=1$ supersymmetry only.

\footnotetext{
${ }^{2}$ Recently, the expression (3.30) has been derived by the direct quantum calculation [12].
} 


\section{Summary}

In this paper we have found the realization of $R$-symmetry for $4 D, \mathcal{N}=4$ and $5 D, \mathcal{N}=2$ supersymmetric gauge theories as the superfield transformations in the relevant harmonic superspaces. The $R$-symmetry transformations were defined in the explicit form, and they mix the gauge multiplet and hypermultiplet harmonic superfields with each other. It was proved that the microscopic actions of $4 D, \mathcal{N}=4$ and $5 D, \mathcal{N}=2 \mathrm{SYM}$ theories are invariant under these transformations without any on-shell conditions on the superfields involved. Thus, the above transformations constitute an additional invariance of $4 D, \mathcal{N}=4$ and $5 D, \mathcal{N}=2$ supersymmetric gauge theories.

The algebraic structure of the harmonic superfield $R$-symmetry transformations was studied. First, these transformations form the closed algebra only on shell. Second, the $R$-symmetry transformations are consistent with both manifest and hidden supersymmetry transformations, which are a necessary element of the harmonic superspace formulations of the maximally extended SYM theories. To be more precise, the $R$-symmetry transformations form a closed algebra with the manifest and hidden supersymmetry transformations. This means, in particular, that the $R$-symmetry transformations and the hidden supersymmetry transformations are in a sense interchangeable. If a manifestly invariant superfield functional is invariant under the $R$-symmetry transformations, then it will be automatically invariant under the hidden supersymmetry transformations and vise versa.

The $R$-symmetry transformations were applied to the problem of the hypermultiplet completion of the low-energy effective action of $4 D, \mathcal{N}=4$ and $5 D, \mathcal{N}=2$ SYM theories, proceeding from the low-energy effective actions in the gauge multiplet sector. Using these transformations, we constructed the leading low-energy complete effective actions for the theories just mentioned, beginning with the terms containing only the gauge multiplet contributions. We have shown that the hypermultiplet dependence of the effective actions under consideration is completely specified by the requirement of invariance under the $R$-symmetry transformations. We focused on the case of $\mathrm{SU}(2)$ gauge group spontaneously broken to $\mathrm{U}(1)$. A generalization to other gauge groups is straightforward. An interesting property is that the effective action is not only invariant under the $R$-symmetry transformations but can be fixed by them up to an overall constant.

It would be tempting to reveal other possible implications of hidden $R$-symmetry in extended superfield gauge theories in diverse dimensions. The maximally supersymmetric gauge theory in six-dimension is $\mathcal{N}=(1,1)$ SYM theory. It has only manifest linear $\mathrm{SU}(2)_{R} \times \mathrm{SU}(2)_{\mathrm{PG}} R$-symmetry and for this reason the methods of the present paper seem not to be appropriate for analysis of the structure of the quantum effective action of this theory in the $\mathcal{N}=(1,0)$ harmonic superspace formulation. Only the considerations based on the hidden $\mathcal{N}=(0,1)$ supersymmetry prove to be adequate [13, 14]. On the other hand, the hidden $R$-symmetry method could be useful in the harmonic superspace formulations of $6 D, \mathcal{N}=(2,0)$ tensor multiplet (see, e.g., [15]). Indeed, only $\mathrm{SU}(2)_{R} R$ symmetry is manifest there, while the rest of the full USp(4) $R$-symmetry of $6 D, \mathcal{N}=(2,0)$ supersymmetry should be realized as a hidden symmetry. We plan to consider this and some other additional examples of exploiting superfield hidden $R$-symmetries elsewhere. 


\section{Acknowledgments}

I.L.B. and E.A.I. are grateful to RFBR grant, project No. 18-02-01046, for a partial support.

\section{A Commutators of $R$-symmetry transformations in $4 D, \mathcal{N}=4 \mathrm{SYM}$ theory}

In this section we directly calculate the commutator of $R$-symmetry with itself for $4 D$, $\mathcal{N}=4$ SYM theory $(2.21)$.

Let us start with the transformation of $\bar{W}$

$$
\left(\delta_{\lambda_{1}} \delta_{\lambda_{2}}-\delta_{\lambda_{2}} \delta_{\lambda_{1}}\right) \bar{W}=\frac{1}{2} \delta_{\lambda_{1}}\left[\lambda_{2}^{-a} q_{a}^{+}-\lambda_{2}^{+a} q_{a}^{-}-\left(\lambda_{2}^{-a} \theta^{+\alpha}-\lambda_{2}^{+a} \theta^{-\alpha}\right) D_{\alpha}^{+} q_{a}^{-}\right]-\left(\lambda_{1} \leftrightarrow \lambda_{2}\right) .
$$

The right-hand side of this relation can further be worked out as

$$
\begin{aligned}
& \frac{1}{8}\left[\lambda_{2}^{-a}\left[\lambda_{1 a}^{+} W+\left(\lambda_{1 a}^{-} \theta^{+\alpha}-\lambda_{1 a}^{+} \theta^{-\alpha}\right) D_{\alpha}^{+} W+\bar{\lambda}_{1 a}^{+} \bar{W}+\left(\bar{\lambda}_{1 a}^{-} \bar{\theta}^{+\dot{\alpha}}-\bar{\lambda}_{1 a}^{+} \bar{\theta}^{-\dot{\alpha}}\right) \bar{D}_{\dot{\alpha}}^{+} \bar{W}\right]\right. \\
& -\lambda_{2}^{+a}\left[\lambda_{1 a}^{-} W+\left(\lambda_{1 a}^{-} \theta^{+\alpha}-\lambda_{1 a}^{+} \theta^{-\alpha}\right) D_{\alpha}^{-} W+\bar{\lambda}_{1 a}^{-} \bar{W}+\left(\bar{\lambda}_{1 a}^{-} \bar{\theta}^{+\dot{\alpha}}-\bar{\lambda}_{1 a}^{+} \bar{\theta}^{-\dot{\alpha}}\right) \bar{D}_{\dot{\alpha}}^{-} \bar{W}\right] \\
& +\left(\lambda_{2}^{-a} \theta^{+\alpha}-\lambda_{2}^{+a} \theta^{-\alpha}\right)\left[\lambda_{1 a}^{-} D_{\alpha}^{+} W-\lambda_{1 a}^{+} D_{\alpha}^{-} W-\left(\lambda_{1 a}^{-} \theta^{+\beta}-\lambda_{1 a}^{+} \theta^{-\beta}\right) D_{\alpha}^{+} D_{\beta}^{-} W\right. \\
& \left.\left.-\left(\bar{\lambda}_{1 a}^{-} \bar{\theta}^{+\dot{\alpha}}-\bar{\lambda}_{1 a}^{+} \bar{\theta}^{-\dot{\alpha}}\right) D_{\alpha}^{+} \bar{D}_{\dot{\alpha}}^{-} \bar{W}\right]\right]-\left(\lambda_{1} \leftrightarrow \lambda_{2}\right) .
\end{aligned}
$$

To properly transform this expression, we note first that the full coefficient before $D_{\alpha}^{+} D_{\beta}^{-} W$ in the next-to-last line in (A.2) is proportional to $\epsilon^{\alpha \beta}$, while $D^{+\alpha} D_{\alpha}^{-} W=0$, as follows from the equation of motion (2.16) for $W$ and the constraints (2.19). Analogously, using the relations (2.18), we can replace $D_{\alpha}^{+} \bar{D}_{\dot{\alpha}}^{-} \bar{W}$ in the last line with $-2 i \partial_{\alpha \dot{\alpha}} \bar{W}$. In addition, we introduce the notations

$$
\lambda^{i j}=\lambda_{2}^{(i a} \bar{\lambda}_{1 a}^{j)}-\lambda_{1}^{(i a} \bar{\lambda}_{2 a}^{j)}, \quad \lambda=\lambda_{2}^{i a} \bar{\lambda}_{1 i a}-\lambda_{1}^{i a} \bar{\lambda}_{2 i a},
$$

where $\lambda^{i j}$ refer to $\mathrm{SU}(2)_{R}$ transformations and $\lambda(\bar{\lambda}=-\lambda)$ to $\mathrm{U}(1)_{R}$ transformations. As a result we obtain

$$
\begin{aligned}
\left(\delta_{\lambda_{1}} \delta_{\lambda_{2}}-\delta_{\lambda_{2}} \delta_{\lambda_{1}}\right) \bar{W}= & \frac{1}{8}\left[-\lambda \bar{W}+\frac{1}{2} \lambda \bar{\theta}^{-\dot{\alpha}} \bar{D}_{\dot{\alpha}}^{+} \bar{W}-\frac{1}{2} \lambda \bar{\theta}^{+\dot{\alpha}} \bar{D}_{\dot{\alpha}}^{-} \bar{W}-\frac{1}{2} \lambda \theta^{+\dot{\alpha}} D_{\alpha}^{-} \bar{W}\right. \\
& -2 i \lambda\left(\theta^{+\alpha} \bar{\theta}^{-\dot{\alpha}}-\theta^{-\alpha} \bar{\theta}^{+\dot{\alpha}}\right) \partial_{\alpha \dot{\alpha}} \bar{W} \\
& +\left(\lambda^{--} \bar{\theta}^{+\dot{\alpha}}-\lambda^{-+} \bar{\theta}^{-\dot{\alpha}}\right) \bar{D}_{\dot{\alpha}}^{+} \bar{W}-\left(\lambda^{+-} \bar{\theta}^{+\dot{\alpha}}-\lambda^{++} \bar{\theta}^{-\dot{\alpha}}\right) \bar{D}_{\dot{\alpha}}^{-} \bar{W} \\
& -\left(\lambda^{+-} \theta^{+\alpha}-\lambda^{++} \theta^{-\alpha}\right) D_{\alpha}^{-} \bar{W} \\
& \left.-2 i\left(\lambda^{--} \theta^{+\alpha} \bar{\theta}^{+\dot{\alpha}}-\lambda^{-+} \theta^{+\alpha} \bar{\theta}^{-\dot{\alpha}}-\lambda^{+-} \theta^{-\alpha} \bar{\theta}^{+\dot{\alpha}}+\lambda^{++} \theta^{-\alpha} \bar{\theta}^{-\dot{\alpha}}\right) \partial_{\alpha \dot{\alpha}} \bar{W}\right] \\
= & \frac{1}{8}\left[\frac{1}{2} \lambda \bar{\theta}^{-\dot{\alpha}} \frac{\partial}{\partial \bar{\theta}^{-\dot{\alpha}}} \bar{W}+\frac{1}{2} \lambda \bar{\theta}^{+\dot{\alpha}} \frac{\partial}{\partial \bar{\theta}^{+\dot{\alpha}}} \bar{W}-\frac{1}{2} \lambda \theta^{+\alpha} \frac{\partial}{\partial \theta^{+\alpha}} \bar{W}\right. \\
& +\left(\lambda^{--} \bar{\theta}^{+\dot{\alpha}}-\lambda^{-+} \bar{\theta}^{-\dot{\alpha}}\right) \frac{\partial}{\partial \bar{\theta}^{-\dot{\alpha}}} \bar{W}+\left(\lambda^{+-} \bar{\theta}^{+\dot{\alpha}}-\lambda^{++} \bar{\theta}^{-\dot{\alpha}}\right) \frac{\partial}{\partial \bar{\theta}^{+\dot{\alpha}} \bar{W}} \\
& +\left(\lambda^{+-} \theta^{+\alpha}-\lambda^{++} \theta^{-\alpha}\right) \frac{\partial}{\partial \theta^{+\alpha}} \bar{W} \\
& \left.-2 i\left(\lambda^{--} \theta^{+\alpha} \bar{\theta}^{+\dot{\alpha}}-\lambda^{-+} \theta^{+\alpha} \bar{\theta}^{-\dot{\alpha}}+\lambda^{+-} \theta^{+\alpha} \bar{\theta}^{-\dot{\alpha}}-\lambda^{++} \theta^{-\alpha} \bar{\theta}^{-\dot{\alpha}}\right) \partial_{\alpha \dot{\alpha}} \bar{W}\right] .
\end{aligned}
$$


Due to the relations (2.19), this expression can be cast in the form

$$
\begin{aligned}
\left(\delta_{\lambda_{1}} \delta_{\lambda_{2}}-\delta_{\lambda_{2}} \delta_{\lambda_{1}}\right) \bar{W}= & -\frac{1}{8} \lambda_{j}^{i}\left(u_{i}^{+} \frac{\partial}{\partial u^{+j}}+u_{i}^{-} \frac{\partial}{\partial u^{-j}}\right) \bar{W} \\
& +\frac{1}{8}\left[\frac{1}{2} \lambda \bar{\theta}^{-\dot{\alpha}} \frac{\partial}{\partial \bar{\theta}^{-\dot{\alpha}}} \bar{W}+\frac{1}{2} \lambda \bar{\theta}^{+\dot{\alpha}} \frac{\partial}{\partial \bar{\theta}^{+\dot{\alpha}}} \bar{W}+\frac{1}{2} \bar{\lambda} \theta^{+\alpha} \frac{\partial}{\partial \theta^{+\alpha}} \bar{W}-\lambda \bar{W}\right] .
\end{aligned}
$$

The transformation law of $W$ can be obtained through the complex conjugation.

Next, we pass to the transformation of $q_{a}^{+}$

$$
\begin{aligned}
\left(\delta_{\lambda_{1}} \delta_{\lambda_{2}}-\delta_{\lambda_{2}} \delta_{\lambda_{1}}\right) q_{a}^{+}= & \frac{1}{2} \delta_{\lambda_{1}}\left[\lambda_{2 a}^{+} W+\left(\lambda_{2 a}^{-} \theta^{+\alpha}-\lambda_{2 a}^{+} \theta^{-\alpha}\right) D_{\alpha}^{+} W\right. \\
& \left.+\bar{\lambda}_{2 a}^{+} \bar{W}+\left(\bar{\lambda}_{2 a}^{-} \bar{\theta}^{+\dot{\alpha}}-\bar{\lambda}_{2 a}^{+} \bar{\theta}^{-\dot{\alpha}}\right) \bar{D}_{\dot{\alpha}}^{+} \bar{W}\right]-\left(\lambda_{1} \leftrightarrow \lambda_{2}\right) \\
= & \frac{1}{8}\left[\lambda_{2 a}^{+}\left[\bar{\lambda}_{1}^{-b} q_{b}^{+}-\bar{\lambda}_{1}^{+b} q_{b}^{-}-\left(\bar{\lambda}_{1}^{-b} \bar{\theta}^{+\dot{\alpha}}-\bar{\lambda}_{1}^{+b} \bar{\theta}^{-\dot{\alpha}}\right) \bar{D}_{\dot{\alpha}}^{+} q_{b}^{-}\right]\right. \\
& +\bar{\lambda}_{2 a}^{+}\left[\lambda_{1}^{-b} q_{b}^{+}-\lambda_{1}^{+b} q_{b}^{-}-\left(\lambda_{1}^{-b} \theta^{+\alpha}-\lambda_{1}^{+b} \theta^{-\alpha}\right) D_{\alpha}^{+} q_{b}^{-}\right] \\
& +\left(\lambda_{2 a}^{-} \theta^{+\alpha}-\lambda_{2 a}^{+} \theta^{-\alpha}\right)\left[-\bar{\lambda}_{1}^{+b} D_{\alpha}^{+} q_{b}^{-}+\left(\bar{\lambda}_{1}^{-b} \bar{\theta}^{+\dot{\alpha}}-\bar{\lambda}_{1}^{+b} \bar{\theta}^{-\dot{\alpha}}\right) D_{\alpha}^{+} \bar{D}_{\dot{\alpha}}^{+} q_{b}^{-}\right] \\
& \left.+\left(\bar{\lambda}_{2 a}^{-} \bar{\theta}^{+\dot{\alpha}}-\bar{\lambda}_{2 a}^{+} \bar{\theta}^{-\dot{\alpha}}\right)\left[-\lambda_{1}^{+b} \bar{D}_{\dot{\alpha}}^{+} q_{b}^{-}+\left(\lambda_{1}^{-b} \theta^{+\alpha}-\lambda_{1}^{+b} \theta^{-\alpha}\right) \bar{D}_{\dot{\alpha}}^{+} D_{\alpha}^{+} q_{b}^{-}\right]\right] \\
& -\left(\lambda_{1} \leftrightarrow \lambda_{2}\right) .
\end{aligned}
$$

Thanks to the analyticity of $q_{a}^{+}$and the equation of motion (2.16), one can replace $\bar{D}_{\dot{\alpha}}^{+} D_{\alpha}^{+} q_{b}^{-}$ in the last line of (A.6) with $-2 i \partial_{\alpha \dot{\alpha}} q_{b}^{+}$, and similarly for $D_{\alpha}^{+} \bar{D}_{\dot{\alpha}}^{+} q_{b}^{-}$. The Lie bracket parameter

$$
\lambda_{(\mathrm{PG})}^{a b}=\lambda_{2}^{i(a} \bar{\lambda}_{1 i}^{b)}-\lambda_{1}^{i(a} \bar{\lambda}_{2 i}^{b)}
$$

is just associated with the $\mathrm{SU}(2)_{\mathrm{PG}}$ symmetry transformations. Therefore,

$$
\begin{aligned}
& \left(\delta_{\lambda_{1}} \delta_{\lambda_{2}}-\delta_{\lambda_{2}} \delta_{\lambda_{1}}\right) q_{a}^{+} \\
& =\frac{1}{8}\left[\lambda_{(\mathrm{PG}) a}^{b} q_{b}^{+}-\lambda^{+-} q_{a}^{+}+\lambda^{++} q_{a}^{-}+\frac{1}{2} \lambda \bar{\theta}^{+\dot{\alpha}} \frac{\partial}{\partial \bar{\theta}^{+\dot{\alpha}}} q_{a}^{+}-\frac{1}{2} \lambda \theta^{+\alpha} \frac{\partial}{\partial \theta^{+\alpha}} q_{a}^{+}\right. \\
& \quad+\left(\lambda^{+-} \bar{\theta}^{+\dot{\alpha}}-\lambda^{++} \bar{\theta}^{-\dot{\alpha}}\right) \frac{\partial}{\partial \bar{\theta}^{+\dot{\alpha}}} q_{a}^{+}+\left(\lambda^{-+} \theta^{+\alpha}-\lambda^{++} \theta^{-\alpha}\right) \frac{\partial}{\partial \theta^{+\alpha}} q_{a}^{+} \\
& \left.\quad+2 i\left(-\lambda^{--} \theta^{+\alpha} \bar{\theta}^{+\dot{\alpha}}+\lambda^{+-} \theta^{-\alpha} \bar{\theta}^{+\dot{\alpha}}+\lambda^{-+} \theta^{+\alpha} \bar{\theta}^{-\dot{\alpha}}-\lambda^{++} \theta^{-\alpha} \bar{\theta}^{-\dot{\alpha}}\right) \partial_{\alpha \dot{\alpha}} q_{a}^{+}\right] .
\end{aligned}
$$

Using the equation of motions (2.16), this expression can be brought to the form

$$
\begin{aligned}
\left(\delta_{\lambda_{1}} \delta_{\lambda_{2}}-\delta_{\lambda_{2}} \delta_{\lambda_{1}}\right) q_{a}^{+}= & \frac{1}{8}\left[\lambda_{(\mathrm{RG}) a}^{b} q_{b}^{+}+\frac{1}{2} \lambda \bar{\theta}^{+\dot{\alpha}} \frac{\partial}{\partial \bar{\theta}^{+\dot{\alpha}}} q_{a}^{+}+\frac{1}{2} \bar{\lambda} \theta^{+\alpha} \frac{\partial}{\partial \theta^{+\alpha}} q_{a}^{+}\right] \\
& -\frac{1}{8} \lambda_{j}^{i}\left(u_{i}^{+} \frac{\partial}{\partial u^{+j}}+u_{i}^{-} \frac{\partial}{\partial u^{-j}}\right) q_{a}^{+}
\end{aligned}
$$

where $\lambda$ is the bracket parameter for $\mathrm{U}(1)_{R}$ transformations. 


\section{B Commutators of $R$-symmetry transformations in $5 D, \mathcal{N}=2 \mathrm{SYM}$ theory}

In this section we calculate the commutator of $R$-symmetry with itself for $5 D, \mathcal{N}=2 \mathrm{SYM}$ theory (3.12).

We start with the transformation of $W$

$$
\begin{aligned}
\left(\delta_{\lambda_{1}} \delta_{\lambda_{2}}-\delta_{\lambda_{2}} \delta_{\lambda_{1}}\right) W= & \frac{1}{4} \delta_{\lambda_{1}}\left[2\left(\lambda_{2}^{+a} q_{a}^{-}-\lambda_{2}^{-a} q_{a}^{+}\right)+\left(\lambda_{2}^{-a} \theta^{+\hat{\alpha}}-\lambda_{2}^{+a} \theta^{-\hat{\alpha}}\right) D_{\hat{\alpha}}^{+} q_{a}^{-}\right] \\
& -\left(\lambda_{1} \leftrightarrow \lambda_{2}\right) .
\end{aligned}
$$

Its right-hand side is evaluated to be

$$
\begin{aligned}
-\frac{1}{8} & {\left[-\left(\lambda_{2}^{+a} \lambda_{1 a}^{+}-\lambda_{1}^{+a} \lambda_{2 a}^{+}\right) \theta^{-\hat{\alpha}} D_{\hat{\alpha}}^{-} W+\left(\lambda_{2}^{+a} \lambda_{1 a}^{-}-\lambda_{1}^{+a} \lambda_{2 a}^{-}\right) \theta^{+\hat{\alpha}} D_{\hat{\alpha}}^{-} W\right.} \\
& +\left(\lambda_{2}^{-a} \lambda_{1 a}^{+}-\lambda_{1}^{-a} \lambda_{2 a}^{+}\right) \theta^{-\hat{\alpha}} D_{\hat{\alpha}}^{+} W-\left(\lambda_{2}^{-a} \lambda_{1 a}^{-}-\lambda_{1}^{-a} \lambda_{2 a}^{-}\right) \theta^{+\hat{\alpha}} D_{\hat{\alpha}}^{+} W \\
& +\left[\left(\lambda_{2}^{-a} \theta^{+\hat{\alpha}}-\lambda_{2}^{+a} \theta^{-\hat{\alpha}}\right)\left(\lambda_{1 a}^{+} \theta^{-\hat{\beta}}-\lambda_{1 a}^{-} \theta^{+\hat{\beta}}\right)\right. \\
& \left.\left.-\left(\lambda_{1}^{-a} \theta^{+\hat{\alpha}}-\lambda_{1}^{+a} \theta^{-\hat{\alpha}}\right)\left(\lambda_{2 a}^{+} \theta^{-\hat{\beta}}-\lambda_{2 a}^{-} \theta^{+\hat{\beta}}\right)\right] D_{\hat{\alpha}}^{+} D_{\hat{\beta}}^{-} W\right] .
\end{aligned}
$$

To bring this expression to a simpler form, we make use of the relation

$$
\left(D_{\hat{\alpha}}^{+} D_{\hat{\beta}}^{-}+D_{\hat{\alpha}}^{-} D_{\hat{\beta}}^{+}\right) W=0
$$

which follows from the equation of motion (3.9) for $W$, the constraint (3.10) and the definition (3.2) of $W{ }^{3}$ Using this relation, we can replace $D_{\hat{\alpha}}^{+} D_{\hat{\beta}}^{-}$with $i \partial_{\hat{\alpha} \hat{\beta}}$ in the last line of (B.2) due to the antisymmetry of the full factor in front of it with respect to the indices $\hat{\alpha}$ and $\hat{\beta}$. We also introduced the notation

$$
\lambda^{i j}=\lambda_{2}^{i a} \lambda_{1 a}^{j}-\lambda_{1}^{i a} \lambda_{2 a}^{j} .
$$

Rewriting eq. (B.2) and substituting the explicit expressions for $D_{\hat{\alpha}}^{-}$and $D_{\hat{\alpha}}^{+}$, we obtain

$$
\begin{aligned}
&\left(\delta_{\lambda_{1}} \delta_{\lambda_{2}}-\delta_{\lambda_{2}} \delta_{\lambda_{1}}\right) W \\
&=-\frac{1}{8}\left[\left(\lambda^{++} \theta^{-\hat{\alpha}}-\lambda^{+-} \theta^{+\hat{\alpha}}\right) \frac{\partial W}{\partial \theta^{+\hat{\alpha}}}+2 i\left(\lambda^{++} \theta^{-\hat{\alpha}}-\lambda^{+-} \theta^{+\hat{\alpha}}\right) \theta^{-\hat{\gamma}} \partial_{\hat{\alpha} \hat{\gamma}} W\right. \\
& \quad+\left(\lambda^{-+} \theta^{-\hat{\alpha}}-\lambda^{--} \theta^{+\hat{\alpha}}\right) \frac{\partial W}{\partial \theta^{-\hat{\alpha}}} \\
&\left.\quad+\left(i \lambda^{-+} \theta^{+\hat{\alpha}} \theta^{-\hat{\beta}}+i \lambda^{+-} \theta^{-\hat{\alpha}} \theta^{+\hat{\beta}}-i \lambda^{--} \theta^{+\hat{\alpha}} \theta^{+\hat{\beta}}-i \lambda^{++} \theta^{-\hat{\alpha}} \theta^{-\hat{\beta}}\right) \partial_{\hat{\alpha} \hat{\beta}} W\right] \\
&=-\frac{1}{8}\left[\left(\lambda^{++} \theta^{-\hat{\alpha}}-\lambda^{+-} \theta^{+\hat{\alpha}}\right) \frac{\partial W}{\partial \theta^{+\hat{\alpha}}}+\left(\lambda^{-+} \theta^{-\hat{\alpha}}-\lambda^{--} \theta^{+\hat{\alpha}}\right) \frac{\partial W}{\partial \theta^{-\hat{\alpha}}}\right. \\
&\left.+i\left(\lambda^{-+} \theta^{-\hat{\alpha}}-\lambda^{--} \theta^{+\hat{\alpha}}\right) \theta^{+\hat{\beta}} \partial_{\hat{\alpha} \hat{\beta}} W+i\left(\lambda^{++} \theta^{-\hat{\alpha}}-\lambda^{+-} \theta^{+\hat{\alpha}}\right) \theta^{-\hat{\beta}} \partial_{\hat{\alpha} \hat{\beta}} W\right] \\
&=-\frac{1}{8} \lambda_{j}^{i}\left(u_{i}^{+} \frac{\partial}{\partial u_{j}^{+}}+u_{i}^{-} \frac{\partial}{\partial u_{j}^{-}}\right) W .
\end{aligned}
$$

When passing to the last line, we exploited the relations (3.10).

\footnotetext{
${ }^{3}$ One also needs to use the identity $\Omega_{\hat{\alpha} \hat{\beta}}=\frac{1}{2} \varepsilon_{\hat{\alpha} \hat{\beta} \hat{\gamma} \hat{\nu}} \Omega^{\hat{\gamma} \hat{\nu}}$.
} 
Now we proceed to the transformations of $q_{a}^{+}$,

$$
\begin{aligned}
\left(\delta_{\lambda_{1}} \delta_{\lambda_{2}}-\delta_{\lambda_{2}} \delta_{\lambda_{1}}\right) q_{a}^{+}=-\frac{1}{2} \delta_{\lambda_{1}}\left[\lambda_{2 a}^{+} W-\lambda_{2 a}^{+} \theta^{-\hat{\alpha}} D_{\hat{\alpha}}^{+} W+\lambda_{a}^{-} \theta^{+\hat{\alpha}} D_{\hat{\alpha}}^{+} W\right]-\left(\lambda_{1} \leftrightarrow \lambda_{2}\right) \\
=-\frac{1}{8}\left[\lambda_{2 a}^{+}\left(2 \lambda_{1}^{+b} q_{b}^{-}-2 \lambda_{1}^{-b} q_{b}^{+}+\lambda_{1}^{-b} \theta^{+\hat{\alpha}} D_{\hat{\alpha}}^{+} q_{b}^{-}-\lambda_{1}^{+b} \theta^{-\hat{\alpha}} D_{\hat{\alpha}}^{+} q_{b}^{-}\right)\right. \\
\left.-\left(\lambda_{2 a}^{+} \theta^{-\hat{\alpha}}-\lambda_{2 a}^{-} \theta^{+\hat{\alpha}}\right) D_{\hat{\alpha}}^{+}\left(2 \lambda_{1}^{+b} q_{b}^{-}+\left(\lambda_{1}^{-b} \theta^{+\hat{\beta}}-\lambda_{1}^{+b} \theta^{-\hat{\beta}}\right) D_{\hat{\beta}}^{+} q_{b}^{-}\right)\right]-\left(\lambda_{1} \leftrightarrow \lambda_{2}\right) \\
=-\frac{1}{8}\left[-\left(\lambda_{2 a}^{i} \lambda_{1 i}^{b}-\lambda_{1 a}^{i} \lambda_{2 i}^{b}\right) q_{b}^{+}-\left(\lambda_{2}^{+b} \lambda_{1 b}^{+}-\lambda_{1}^{+b} \lambda_{2 b}^{+}\right) q_{a}^{-}+\left(\lambda_{2}^{+b} \lambda_{1 b}^{-}-\lambda_{1}^{+b} \lambda_{2 b}^{-}\right) q_{a}^{+}\right. \\
-\lambda_{2}^{+b}\left(\lambda_{1 b}^{+} \theta^{-\hat{\alpha}}-\lambda_{1 b}^{-} \theta^{+\hat{\alpha}}\right) D_{\hat{\alpha}}^{-} q_{a}^{+}+\lambda_{1}^{+b}\left(\lambda_{2 b}^{+} \theta^{-\hat{\alpha}}-\lambda_{2 b}^{-} \theta^{+\hat{\alpha}}\right) D_{\hat{\alpha}}^{-} q_{a}^{+} \\
\left.-2 i\left(\lambda_{2}^{+b} \theta^{-\hat{\alpha}}+\lambda_{2}^{-b} \theta^{+\hat{\alpha}}\right)\left(\lambda_{1 b}^{+} \theta^{-\hat{\beta}}-\lambda_{1 b}^{-} \theta^{+\hat{\beta}}\right) \partial_{\hat{\alpha} \hat{\beta}} q_{a}^{+}\right]
\end{aligned}
$$

where the relations $D_{\hat{\alpha}}^{-} q^{+a}=-D_{\hat{\alpha}}^{+} q^{-a}$ and $D_{\hat{\alpha}}^{+} D_{\hat{\beta}}^{+} q^{-a}=-2 i \partial_{\hat{\alpha} \hat{\beta}} q^{+a}$ were used.

Substituting the explicit expressions for $D_{\hat{\alpha}}^{-}$, we finally obtain

$$
\begin{aligned}
&\left(\delta_{\lambda_{1}} \delta_{\lambda_{2}}-\delta_{\lambda_{2}} \delta_{\lambda_{1}}\right) q_{a}^{+} \\
&=-\frac{1}{8}\left[-\left(\lambda_{2 a}^{i} \lambda_{1 i}^{b}-\lambda_{1 a}^{i} \lambda_{2 i}^{b}\right) q_{b}^{+}-\left(\lambda_{2}^{+b} \lambda_{1 b}^{+}-\lambda_{1}^{+b} \lambda_{2 b}^{+}\right) q_{a}^{-}+\left(\lambda_{2}^{+b} \lambda_{1 b}^{-}-\lambda_{1}^{+b} \lambda_{2 b}^{-}\right) q_{a}^{+}\right. \\
&+\lambda_{2}^{+b}\left(\lambda_{1 b}^{+} \theta^{-\hat{\alpha}}-\lambda_{1 b}^{-} \theta^{+\hat{\alpha}}\right) \frac{\partial q_{a}^{+}}{\partial \theta^{+\hat{\alpha}}}-\lambda_{1}^{+b}\left(\lambda_{2 b}^{+} \theta^{-\hat{\alpha}}-\lambda_{2 b}^{-} \theta^{+\hat{\alpha}}\right) \frac{\partial q_{a}^{+}}{\partial \theta^{+\hat{\alpha}}} \\
&\left.-2 i \lambda_{1}^{+b} \lambda_{2 b}^{+} \theta^{-\hat{\alpha}} \theta^{-\hat{\beta}} \partial_{\hat{\alpha} \hat{\beta}} q_{a}^{+}-2 i \lambda_{1}^{-b} \lambda_{2 b}^{-} \theta^{+\hat{\alpha}} \theta^{+\hat{\beta}} \partial_{\hat{\alpha} \hat{\beta}} q_{a}^{+}\right] \\
&=-\frac{1}{8}\left(\lambda_{2}^{i a} \lambda_{1 a j}-\lambda_{1}^{i a} \lambda_{2 a j}\right)\left(u_{i}^{+} \frac{\partial}{\partial u_{j}^{+}}+u_{i}^{-} \frac{\partial}{\partial u_{j}^{-}}\right) q_{a}^{+}+\frac{1}{8}\left(\lambda_{2 a}^{i} \lambda_{1 i}^{b}-\lambda_{1 a}^{i} \lambda_{2 i}^{b}\right) q_{b}^{+} .
\end{aligned}
$$

When passing to the last line, we used the equation of motion (3.9).

Open Access. This article is distributed under the terms of the Creative Commons Attribution License (CC-BY 4.0), which permits any use, distribution and reproduction in any medium, provided the original author(s) and source are credited.

\section{References}

[1] N. Seiberg, Notes on theories with 16 supercharges, Nucl. Phys. Proc. Suppl. 67 (1998) 158 [hep-th/9705117] [INSPIRE].

[2] A. Galperin, E. Ivanov, S. Kalitsyn, V. Ogievetsky and E. Sokatchev, Unconstrained $\mathcal{N}=2$ Matter, Yang-Mills and Supergravity Theories in Harmonic Superspace, Class. Quant. Grav. 1 (1984) 469 [Erratum ibid. 2 (1985) 127] [INSPIRE].

[3] A.S. Galperin, E.A. Ivanov, V.I. Ogievetsky and E.S. Sokatchev, Harmonic Superspace, Cambridge University Press (2001) [INSPIRE].

[4] I.L. Buchbinder, E.A. Ivanov and N.G. Pletnev, Superfield approach to the construction of effective action in quantum field theory with extended supersymmetry, Phys. Part. Nucl. $\mathbf{4 7}$ (2016) 291 [INSPIRE]. 
[5] I.L. Buchbinder, E.A. Ivanov and I.B. Samsonov, The low-energy $\mathcal{N}=4$ SYM effective action in diverse harmonic superspaces, Phys. Part. Nucl. 48 (2017) 333 [arXiv: 1603.02768] [INSPIRE].

[6] I.L. Buchbinder and E.A. Ivanov, Hidden supersymmetry as a key to constructing low-energy superfield effective actions, arXiv:1911.04485 [INSPIRE].

[7] I.L. Buchbinder and E.A. Ivanov, Complete $\mathcal{N}=4$ structure of low-energy effective action in $\mathcal{N}=4$ superYang-Mills theories, Phys. Lett. B 524 (2002) 208 [hep-th/0111062] [INSPIRE].

[8] M. Dine and N. Seiberg, Comments on higher derivative operators in some SUSY field theories, Phys. Lett. B 409 (1997) 239 [hep-th/9705057] [InSPIRE].

[9] I.L. Buchbinder, E.A. Ivanov and I.B. Samsonov, Low-energy effective action in $5 D, \mathcal{N}=2$ supersymmetric gauge theory, Nucl. Phys. B 940 (2019) 54 [arXiv:1812.07206] [InSPIRE].

[10] B.M. Zupnik, The Action of the Supersymmetric $\mathcal{N}=2$ Gauge Theory in Harmonic Superspace, Phys. Lett. B 183 (1987) 175 [InSPIRE].

[11] I.L. Buchbinder and N.G. Pletnev, Effective actions in $\mathcal{N}=1$, D5 supersymmetric gauge theories: harmonic superspace approach, JHEP 11 (2015) 130 [arXiv: 1510.02563] [INSPIRE].

[12] I.L. Buchbinder, E.A. Ivanov and B.S. Merzlikin, Quantum calculation of the low-energy effective action in $5 D, \mathcal{N}=2$ SYM theory, Phys. Lett. B 802 (2020) 135218 [arXiv: 1912.07787] [INSPIRE].

[13] I.L. Buchbinder, E.A. Ivanov and B.S. Merzlikin, Leading low-energy effective action in $6 D$, $\mathcal{N}=(1,1)$ SYM theory, JHEP 09 (2018) 039 [arXiv:1711.03302] [INSPIRE]

[14] I.L. Buchbinder, E.A. Ivanov and B.S. Merzlikin, Low-energy $6 D, \mathcal{N}=(1,1) S Y M$ effective action beyond the leading approximation, Nucl. Phys. B 954 (2020) 114995 [arXiv: 1912.02634] [INSPIRE].

[15] I.L. Buchbinder and N.G. Pletnev, Construction of $6 D$ supersymmetric field models in $\mathcal{N}=(1,0)$ harmonic superspace, Nucl. Phys. B $892(2015) 21$ [arXiv:1411.1848] [InSPIRE]. 